\title{
Dense Packings of Polyhedra: Platonic and Archimedean Solids
}

\author{
S. Torquato ${ }^{1,2,3,4,5,6}$ and Y. Jiao ${ }^{6}$ \\ ${ }^{1}$ Department of Chemistry, Princeton University, \\ Princeton New Jersey 08544, USA \\ ${ }^{2}$ Princeton Center for Theoretical Science, \\ Princeton University, Princeton New Jersey 08544, USA \\ ${ }^{3}$ Princeton Institute for the Science and Technology of Materials, \\ Princeton University, Princeton New Jersey 08544, USA \\ ${ }^{4}$ Program in Applied and Computational Mathematics, \\ Princeton University, Princeton New Jersey 08544, USA \\ ${ }^{5}$ School of Natural Sciences, Institute for Advanced Study, Princeton NJ 08540 and \\ ${ }^{6}$ Department of Mechanical and Aerospace Engineering, \\ Princeton University, Princeton New Jersey 08544, USA
}




\begin{abstract}
Understanding the nature of dense particle packings is a subject of intense research in the physical, mathematical and biological sciences. The preponderance of previous work has focused on spherical particles, and very little is known about dense polyhedral packings. We formulate the problem of generating dense packings of nonoverlapping, non-tiling polyhedra within an adaptive fundamental cell subject to periodic boundary conditions as an optimization problem, which we call the Adaptive Shrinking Cell (ASC) scheme. This novel optimization problem is solved here (using a variety of multi-particle initial configurations) to find the dense packings of each of the Platonic solids in three-dimensional Euclidean space $\mathbb{R}^{3}$, except for the cube, which is the only Platonic solid that tiles space. We find the densest known packings of tetrahedra, icosahedra, dodecahedra, and octahedra with densities $0.823 \ldots, 0.836 \ldots, 0.904 \ldots$, and $0.947 \ldots$, respectively. It is noteworthy that the densest tetrahedral packing possesses no long-range order. Unlike the densest tetrahedral packing, which must not be a Bravais lattice packing, the densest packings of the other non-tiling Platonic solids that we obtain are their previously known optimal (Bravais) lattice packings. We also derive a simple upper bound on the maximal density of packings of congruent nonspherical particles, and apply it to Platonic solids, Archimedean solids, superballs and ellipsoids. Provided that what we term the "asphericity" (ratio of the circumradius to inradius) is sufficiently small, the upper bounds are relatively tight and thus close to the corresponding densities of the optimal lattice packings of the centrally symmetric Platonic and Archimedean solids. Our simulation results, rigorous upper bounds, and other theoretical arguments lead us to the conjecture that the densest packings of Platonic and Archimedean solids with central symmetry are given by their corresponding densest lattice packings. This can be regarded to be the analog of Kepler's sphere conjecture for these solids. The truncated tetrahedron is the only non-centrally symmetric Archimedean solid, the densest known packing of which is a non-lattice packing with density at least as high as $23 / 24=0.958333 \ldots$ We discuss the validity of our conjecture to packings of superballs, prisms and anti-prisms as well as to high-dimensional analogs of the Platonic solids. In addition, we conjecture that the optimal packing of any convex, congruent polyhedron without central symmetry generally is not a lattice packing. Finally, we discuss the possible applications and generalizations of the ASC scheme in the predicting the crystal structures of polyhedral nanoparticles and the study of random packings of hard polyhedra.
\end{abstract}


PACS numbers: 05.20.Jj, 45.70.-n, 61.50.Ah 


\section{INTRODUCTION}

Particle packing problems are ancient, dating back to the dawn of civilization. Bernal has remarked that "heaps" (particle packings) were the first things that were ever measured in the form of basketfuls of grain for the purpose of trading or of collection of taxes [1]. Dense packings of hard particles have served as useful models to understand the structure of liquid, glassy and crystal states of matter [2, 3, 4], granular media [5], and heterogeneous materials [3, 6]. Understanding the symmetries and other mathematical properties of the densest packings in arbitrary dimensions is a problem of long-standing interest in discrete geometry and number theory [7].

A large collection of nonoverlapping solid objects (particles) in $d$-dimensional Euclidean space $\mathbb{R}^{d}$ is called a packing. The packing density $\phi$ is defined as the fraction of space $\mathbb{R}^{d}$ covered by the particles. A problem that has been a source of fascination to mathematicians and scientists for centuries is the determination of the densest arrangement(s) of particles that do not tile space and the associated maximal density $\phi_{\max }$ [7]. Finding the maximal-density packing arrangements is directly relevant to understanding the structure and properties of crystalline equilibrium phases of particle systems as well as their (zero-temperature) ground-state structures in low dimensions in which the interactions are characterized by steep repulsions and short-ranged attractions.

The preponderance of previous investigations have focused on dense packings of spheres in various dimensions [3, 7, 8, 9]. For congruent particles in three dimensions, the sphere is the only non-tiling particle for which the densest packing arrangements can be proved [10]. It is only very recently that attention has turned to finding the maximal-density packing arrangements of nonspherical particles in $\mathbb{R}^{3}$, including ellipsoids [11, 12], tetrahedra [13, 14, 15], and superballs [16, 17]. Very little is known about the densest packings of polyhedral particles [18].

The Platonic solids (mentioned in Plato's Timaeus) are convex polyhedra with faces composed of congruent convex regular polygons. There are exactly five such solids: the tetrahedron (P1), icosahedron (P2), dodecahedron (P3), octahedron (P4), and cube (P5) (see Fig. 11) [19]. One major concern in this paper is the determination of the densest packings of each of the Platonic solids in three-dimensional Euclidean space $\mathbb{R}^{3}$, except for the cube, which is the only Platonic solid that tiles space. 


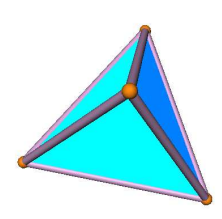

$\mathrm{P} 1$

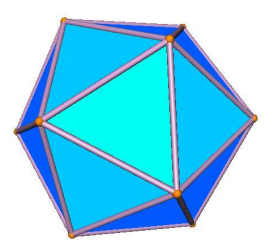

$\mathrm{P} 2$

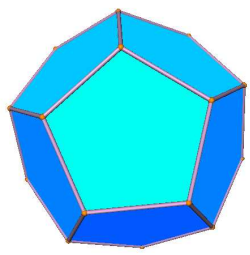

P3

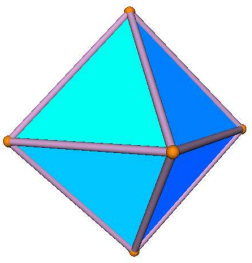

P4

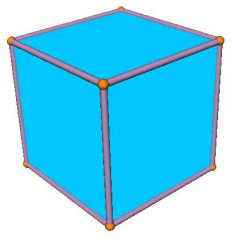

P5

FIG. 1: (color online). The five Platonic solids: tetrahedron (P1), icosahedron (P2), dodecahedron (P3), octahedron (P4), and cube (P5).

It is useful to highlight some basic geometrical properties of the Platonic solids that we will employ in subsequent sections of the paper. The dihedral angle $\theta$ is the interior angle between any two face planes and is given by

$$
\sin \frac{\theta}{2}=\frac{\cos (\pi / q)}{\sin (\pi / p)}
$$

where $p$ is the number of sides of each face and $q$ is the number of faces meeting at each vertex. Thus, $\theta$ is $2 \sin ^{-1}(1 / \sqrt{3}), 2 \sin ^{-1}(\Phi / \sqrt{3}), 2 \sin ^{-1}\left(\Phi / \sqrt{\Phi^{2}+1}\right), 2 \sin ^{-1}(\sqrt{2 / 3})$, and $\pi / 2$, for the tetrahedron, icosahedron, dodecahedron, octahedron, and cube, respectively, where $\Phi=(1+\sqrt{5}) / 2$ is the golden ratio. Thus, since the dihedral angle for the cube is the only one that is a submultiple of $2 \pi$, the cube is the only Platonic solid that tiles space. We note in passing that in addition to the regular tessellation of space by cubes in the simple cubic lattice arrangement, there are an infinite number of other irregular tessellations of space by cubes [20]. Figure 2 shows a portion of a realization of a two-dimensional analog of such an irregular tessellation.

Every polyhedron has a dual polyhedron with faces and vertices interchanged. The dual of each Platonic solid is another Platonic solid, and therefore they can be arranged into dual pairs: the tetrahedron is self-dual (i.e., its dual is another tetrahedron), the icosahedron and dodecahedron form a dual pair, and the octahedron and cube form a dual pair.

An Archimedean solid is a highly symmetric, semi-regular convex polyhedron composed of two or more types of regular polygons meeting in identical vertices. There are thirteen Archimedean solids: truncated tetrahedron (A1), truncated icosahedron (A2), snub cube (A3), snub dodecahedron (A4), rhombicosidodecahdron (A5), truncated icosidodecahdron (A6), truncated cuboctahedron (A7), icosidodecahedron (A8), rhombicuboctahedron (A9), 


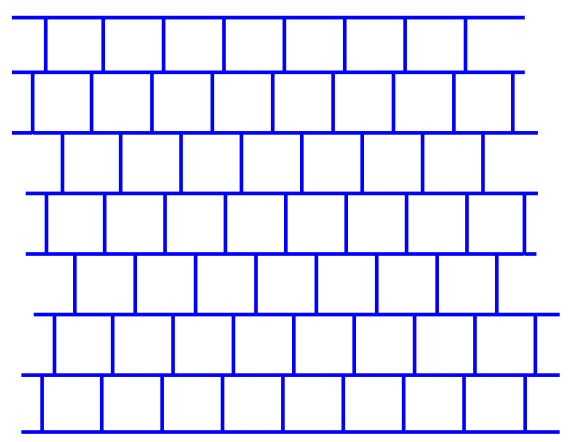

FIG. 2: (color online). A portion of a realization of an irregular tiling of the plane by squares.

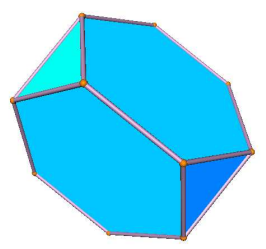

A1

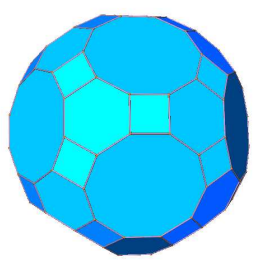

A6

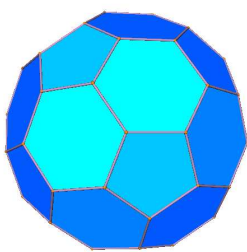

A2

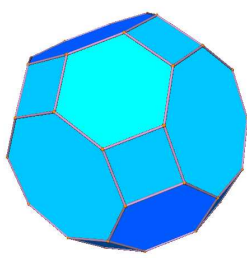

A7

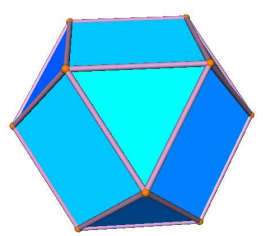

A11

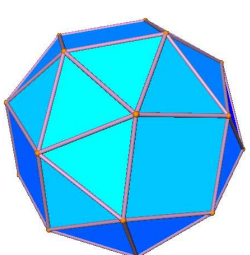

A3

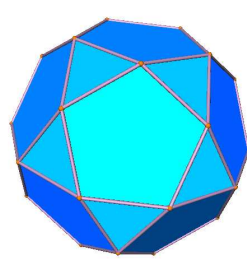

A8

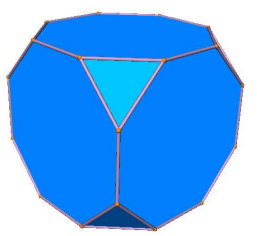

A12

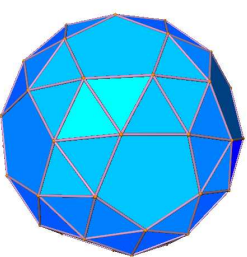

A4

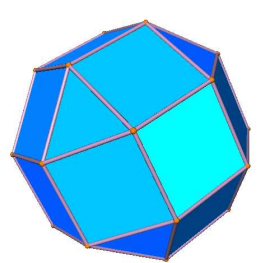

A9

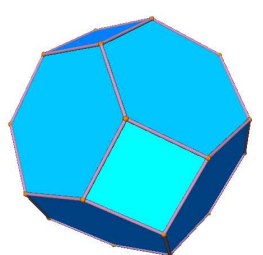

A13

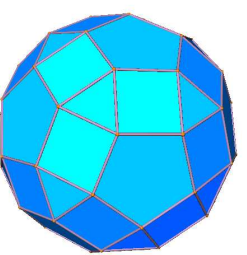

A5

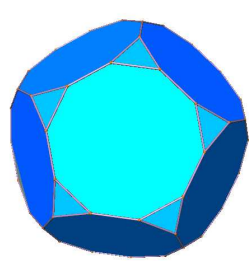

A10

FIG. 3: The 13 Archimedean solids: truncated tetrahedron (A1), truncated icosahedron (A2), snub cube (A3), snub dodecahedron (A4), rhombicosidodecahdron (A5), truncated icosidodecahdron (A6), truncated cuboctahedron (A7), icosidodecahedron (A8), rhombicuboctahedron (A9), truncated dodecahedron (A10), cuboctahedron (A11), truncated cube (A12), and truncated octahedron (A13). 
truncated dodecahedron (A10), cuboctahedron (A11), truncated cube (A12) and truncated octahedron (A13) (see Fig. 31). Note that the truncated octahedron is the only Archimedean solid that tiles space.

Another important observation is that the tetrahedron (P1) and the truncated tetrahedron (A1) are the only Platonic and Archimedean solids, respectively, that are not centrally symmetric. A particle is centrally symmetric if it has a center $C$ that bisects every chord through $C$ connecting any two boundary points of the particle, i.e., the center is a point of inversion symmetry. We will see that the central symmetry of the majority of the Platonic and Archimedean solids (P2 - P5, A2 - A13) distinguish their dense packing arrangements from those of the non-centrally symmetric ones (P1 and A1) in a fundamental way.

Some basic definitions concerning packings are given here. A saturated packing is one in which there is no space available to add another particle to the packing. A lattice $\Lambda$ in $\mathbb{R}^{d}$ is a subgroup consisting of the integer linear combinations of vectors that constitute a basis for $\mathbb{R}^{d}$ [21]. A lattice packing $P_{L}$ is one in which the centroids of the nonoverlapping particles are located at the points of $\Lambda$, each oriented in the same direction. The set of lattice packings is a subset of all possible packings in $\mathbb{R}^{d}$. In a lattice packing, the space $\mathbb{R}^{d}$ can be geometrically divided into identical regions $F$ called fundamental cells, each of which contains just the centroid of one particle. Thus, the density of a lattice packing is given by

$$
\phi=\frac{v_{p}}{\operatorname{Vol}(F)}
$$

where $v_{p}$ is the volume of a $d$-dimensional particle and $\operatorname{Vol}(F)$ is the volume of a fundamental cell.

A more general notion than a lattice packing is a periodic packing. A periodic packing of congruent particles is obtained by placing a fixed nonoverlapping configuration of $N$ particles (where $N \geq 1$ ) with arbitrary orientations in each fundamental cell of a lattice $\Lambda$. Thus, the packing is still periodic under translations by $\Lambda$, but the $N$ particles can occur anywhere in the chosen fundamental cell subject to the nonoverlap condition. The packing density of a periodic packing is given by

$$
\phi=\frac{N v_{p}}{\operatorname{Vol}(F)}=\rho v_{p}
$$

where $\rho=N / \operatorname{Vol}(F)$ is the number density, i.e., the number of particles per unit volume.

The determination of the maximal-density arrangements of non-tiling polyhedral particles is a notoriously difficult problem, especially since such extremal structures will generally 
be non-Bravais-lattice packings. Computer simulations that seek the maximal-density packings can be an indispensable tool, especially if they can incorporate collective motions of the particles in order to obtain, in principle, the highest possible densities. However, the challenge presented by polyhedral particles in $\mathbb{R}^{3}$ is the non-smooth (i.e., nonanalytic) nature of the particle shape. In the case of smoothly-shaped particles, such as spheres, ellipsoids and superballs, one can construct analytic "overlap potential functions" for the particles [24] and hence one can employ efficient collision-driven molecular dynamics (MD) growth packing algorithms that inherently involve collective particle motions [12, 16, 17, 25, 26]. The fact that analytic overlap potential functions cannot be constructed for polyhedral particles prevents us from using event-driven MD growth methods to study such systems.

In this paper, we devise a novel optimization scheme, called the Adaptive Shrinking Cell (ASC), that can be applied to generate dense packings of polyhedra in $\mathbb{R}^{3}$. We employ it specifically to obtain the densest known packings of tetrahedra, icosahedra, dodecahedra, and octahedra with densities $0.823 \ldots, 0.836 \ldots, 0.904 \ldots$, and $0.947 \ldots$, respectively. The result for tetrahedra improves upon the density reported in our recent investigation [27, 28]. Unlike the densest tetrahedral packing, which must not be a Bravais lattice packing [13], the densest packings of the other non-tiling Platonic solids that we obtain are their corresponding densest lattice packings [22, 23].

We also derive a simple upper bound on the maximal density of packings of congruent nonspherical particles, and apply it to Platonic solids, Archimedean solids, superballs and ellipsoids. We introduce the "asphericity" parameter $\gamma$ (ratio of the circumradius to inradius) to show that when $\gamma$ is sufficiently small, the upper bounds are relatively tight and thus close to the corresponding densities of the optimal lattice packings of octahedra, dodecahedra and icosahedra as well as of the majority of the Archimedean solids with central symmetry.

Our simulation results as well as theoretical arguments lead us to conjecture that the densest packings of Platonic and Archimedean solids with central symmetry are given by their corresponding densest lattice packings. This can be regarded to be the analog of Kepler's sphere conjecture for these solids. The truncated tetrahedron is the only noncentrally symmetric Archimedean solid, the densest known packing of which is a non-lattice packing with density as high as $23 / 24=0.958333 \ldots$ O Our work also suggests that the optimal packings of superballs are their corresponding densest lattice packings.

In a recent letter [27], we briefly reported the densest known packings of the non-tiling 
Platonic solids obtained using the ASC algorithm and proposed the aforementioned conjecture concerning the optimal packings of the Platonic and Archimedean solids. In this paper, we expand on theoretical and computational details and report additional new results. In particular, we provide comprehensive details about the ASC scheme and the simulation results (Sec. II), including a discussion about the various initial configurations we used for the ASC algorithm (Sec. III). Moreover, we have improved on the highest tetrahedral packing density reported in Ref. [27] (i.e., from $0.782 \ldots$ to $0.823 \ldots$. ), by exploring a broad range of dynamical parameters for the algorithm and initial configurations. Certain pair statistics of the densest known packing of tetrahedra (e.g., the contact number, the centroidal correlation function and the face-normal correlation function) are given (Sec. III). It is noteworthy that the densest tetrahedral packing is a non-Bravais structure with a complex periodic cell and possesses no long-range order.

The initial configurations for the icosahedral, dodecahedral and octahedral packings are described and the numerical challenges in producing dense packings of such polyhedral particles are discussed (Sec. III). In addition, a detailed derivation of the upper bound and tables containing the geometrical characteristics of the Platonic and Archimedean solids as well as their upper bound values are given (Sec. IV). The upper bound is also applied to superballs and ellipsoids (which was not done in Ref. [27]). Moreover, we provide the major elements of a possible proof of our conjecture (Sec. V). We also discuss how our conjecture could be generalized to other centrally symmetric polyhedral particles such as prisms and anti-prisms as well as high-dimensional analogs of the Platonic solids. Our work also naturally leads to another conjecture reported here for the first time, namely, the optimal packing of any convex, congruent polyhedron without central symmetry is generally not a (Bravais) lattice packing (Sec. V).

Furthermore, we discuss the possible applications and generalizations of the ASC scheme to predict the crystal structures of polyhedral nanoparticles and to the study of random packings of hard polyhedra. Finally, we collect in appendices basic packing characteristics of various optimal lattice and non-lattice packings of polyhedra (including lattice vectors) that have been scattered throughout the literature and provide lattice vectors and other characteristics of the densest known packings of tetrahedra (obtained here) and truncated tetrahedra. 


\section{ADAPTIVE SHRINKING CELL (ASC) OPTIMIZATION SCHEME}

We formulate the problem of generating dense packings of nonoverlapping, non-tiling polyhedra within a fundamental cell in $\mathbb{R}^{3}$ subject to periodic boundary conditions as an optimization problem. In particular, the objective function is taken to be the negative of the packing density $\phi$. Starting from an initial unsaturated packing configuration of particles of fixed size in the fundamental cell, the positions and orientations of the polyhedra are design variables for the optimization. Importantly, we also allow the boundary of the fundamental cell to deform macroscopically as well as compress or expand (while keeping the particles fixed in size) such that there is a net compression (increase of the density of the packing) in the final state [29]. Thus, the deformation and compression/expansion of the cell boundary, which we call the adaptive fundamental cell, are also design variables. We are not aware of any packing algorithm that employs both a sequential search of the configurational space of the particles and the space of lattices via an adaptive fundamental cell that shrinks on average to obtain dense packings. We will call this optimization scheme the Adaptive Shrinking Cell (ASC).

We will see that the ASC optimization scheme allows for some desired collective motions of the particles to find the optimal lattice for the periodic cell. This is to be contrasted with previous treatments that use a fixed shape for the fundamental cell, which may or may not be the optimal shape. Figure 4 illustrates a simple sequence of configuration changes for a four-particle system (which is explained in more detail in Sec. II.A). By efficiently exploring the design-variable space (DVS), which consists of the particle configurational space as well as the space of lattices via an adaptive fundamental cell, the ASC scheme enables one to find a point in the DVS in the neighborhood of the starting point that has a higher packing density than the initial density. The process is continued until the deepest minimum of the objective function (a maximum of packing density) is obtained, which could be either a local or global optimum, depending on the particle shapes.

The ASC optimization problem could be solved using various techniques, depending on the shapes of the particles. For example, for spheres, one can linearize the objective function and the constraints, and we have found that linear programming techniques can efficiently produce optimal solutions for such ASC problems [30]. However, for hard polyhedra the non-overlapping conditions given by the separation axis theorem (discussed in detail below) 


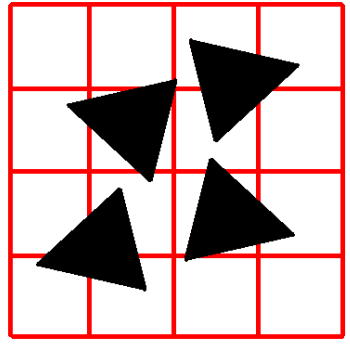

(a)

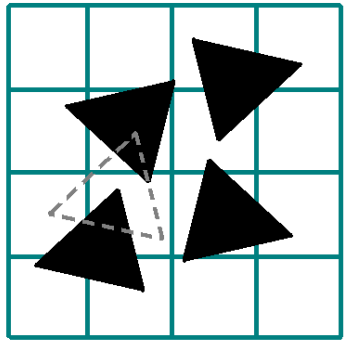

(b)

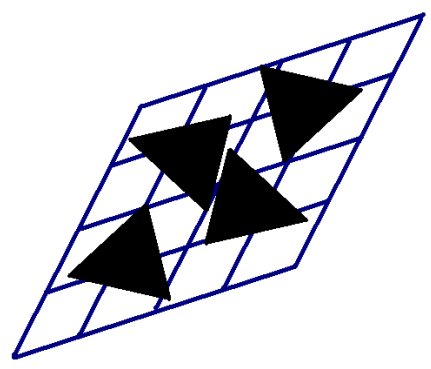

(c)

FIG. 4: (color online). Sequential changes of the packing configuration due to the design variables in the ASC algorithm. (a) An initial configuration of four particles. (b) A trial move of a randomly selected particle that is rejected because it overlaps another particle. (c) A trial move that is accepted, which results in a deformation and compression (small in magnitude) changing the fundamental cell shape and size as well as the relative distances between the particles. The large fundamental cell is divided into smaller sub-cells in order to implement the "cell method" discussed in Sec. II.D.

involve at least quartic inequalities, which makes it inefficient to solve even using nonlinear programming methods.

Here, for polyhedral particles, we solve the ASC optimization problem using a stochastic procedure, i.e., Monte Carlo (MC) method with a Metropolis acceptance rule for trial moves to search the DVS efficiently. However, it is important to distinguish our procedure that incorporates deformation and compression/expansion of the fundamental cell (i.e., the space of lattices) as design variables from previous MC hard-particle packing algorithms [31]. In standard MC simulations, arbitrarily selected individual particle is given random displacement or rotation. This sequential movement method is not able to account for any collective motions of the particles, which is crucial to increasing the packing density, as pointed out in Sec. I. In our procedure, the deformation/compression/expansion of the boundary at least in part allows for collective particle motions in a direction leading to higher packing density. Moreover, it is the overall compression of the fundamental cell that causes the packing density to increase, not the growth of the particles as in most MD and MC hard-particle 
packing algorithms [25, 26, 31].

At first glance, one might surmise that an algorithm that employs particle growth with an adaptive non-shrinking fundamental cell is equivalent to our choice of fixing the particle size while allowing the cell to shrink on average. The former is computationally less efficient than the latter for polyhedral particles. Specifically, growth of polyhedral particles requires manipulating the coordinates of the vertices of each particle, and thus involves at least $d N n_{v}$ numerical operations, where $d$ is the spatial dimension, $N$ is the total number of particles in the system and $n_{v}$ is the number of vertices per particle. It is much more computationally expensive to use growing/shrinking particles as trial moves for the optimization scheme, especially when the number of particles is large, compared to our adaptive fundamental cell approach, which only requires manipulating $d(d+1) / 2$ strain components.

In the ASC scheme, the macroscopic deformation and compression/expansion of the fundamental cell of the lattice is completely specified by a strain tensor. Since we only consider small deformations, linear strain analysis can be applied here. Starting from an initial configuration, a trial configuration can be generated by moving (translating and rotating) a randomly chosen particle or by a random macroscopic deformation and compression/expansion of the fundamental cell. If any two particles overlap, the trial configuration is rejected; otherwise, if the fundamental cell shrinks in size (which makes the density $\phi$ higher), the trial configuration is accepted. On the other hand, if the cell expands in size, the trial configuration is accepted with a specified probability $p_{a c c}$, which decreases as $\phi$ increases and approaches zero when the jamming limit [32] (i.e., locally maximal dense packing) is reached. In particular, we find $p_{a c c}$, with an initial value $p_{a c c} \sim 0.35$, decreasing as a power law with exponent equal to -1 works well for most systems that we studied. The particle motion is equally likely to be a translation or a rotation. The ratio of the number of particle motions to the number of boundary trial moves should be greater than unity (especially towards the end of the simulation), since compressing a dense packing could result in many overlaps between the particles. Depending on the initial density, the magnitudes of the particle motions and strain components (e.g., the dynamical parameters) need to be chosen carefully to avoid the system getting stuck in some shallow local minimum. The parameters should also be adjusted accordingly as the packing density increases, especially towards the jamming point. The number of total Monte-Carlo moves per particle is of the order of $5 \times 10^{6}$. 
In the ensuing subsections, we describe in detail how we implement particle motions as well as the deformation and compression/expansion of the fundamental cell, and precise

check for interparticle overlaps using the separation axis theorem [33]. We also discuss the cell method and nearest-neighbor lists that are employed to speed up the simulations.

\section{A. Particle Motions}

The fact that a polyhedron is the convex hull of its vertices makes the set of vertices a useful geometrical representation of a such particle. It is convenient to choose the origin of the local coordinate system for the vertices to be the centroid of the polyhedron. Other important geometrical properties of the polyhedron, such as its faces and edges, can be represented as certain subsets of the vertices.

Let the Euclidean position of the centroid of the $j$ th particle be $\mathbf{x}_{j}^{E}(j=1, \ldots, N)$. A translational motion of the particle centroid can be obtained by generating a randomly oriented displacement $\Delta \mathbf{x}_{j}^{E}$ with small magnitude $\left(10^{-4}-10^{-6}\right.$ of the characteristic length of the particle), i.e.,

$$
\overline{\mathbf{x}}_{j}^{E}=\mathbf{x}_{j}^{E}+\Delta \mathbf{x}_{j}^{E}
$$

A rotational motion can be generated by rotating the particle (all of its vertices) along a randomly chosen axis (passing through its centroid) by a random small angle $\theta$. Let the vector $\mathbf{v}_{i}$ originating at the centroid denote the vertex $i$. We have

$$
\overline{\mathbf{v}}_{i}=\mathbf{R} \cdot \mathbf{v}_{i}^{\perp}+\mathbf{v}_{i}^{\|}
$$

where $\mathbf{v}_{i}^{\perp}$ and $\mathbf{v}_{i}^{\|}$are the components of $\mathbf{v}_{i}$ perpendicular and parallel to the rotation axis, respectively, and $\mathbf{R}$ is the rotation matrix, i.e.,

$$
\mathbf{R}=\left[\begin{array}{ccc}
\cos \theta & -\sin \theta & 0 \\
\sin \theta & \cos \theta & 0 \\
0 & 0 & 1
\end{array}\right]
$$

If this motion does not result in the overlap with another particle, the trial move is accepted; otherwise it is rejected. 


\section{B. Adaptive Fundamental Cell}

For a lattice-based periodic packing, the fundamental cell is specified by the lattice vectors $\mathbf{a}_{i}(i=1,2,3)$. Recall that the Euclidean coordinates of the particle centroids are $\mathbf{x}_{j}^{E}$ $(j=1, . ., N)$. The relative coordinates of the centroids with respect to the lattice vectors are given by

$$
\mathbf{x}_{j}^{E}=\mathbf{\Gamma} \cdot \mathbf{x}_{j}^{L}
$$

where $\boldsymbol{\Gamma}=\left[\mathbf{a}_{1}, \mathbf{a}_{2}, \mathbf{a}_{3}\right]$.

The adaptive fundamental cell allows for a small strain of the fundamental cell, including both volume and shape changes, which is represented by a symmetric strain tensor $\varepsilon$, i.e.,

$$
\Delta \Gamma=\varepsilon \cdot \Gamma
$$

where

$$
\boldsymbol{\varepsilon}=\left[\begin{array}{lll}
\epsilon_{11} & \epsilon_{12} & \epsilon_{13} \\
\epsilon_{21} & \epsilon_{22} & \epsilon_{23} \\
\epsilon_{31} & \epsilon_{32} & \epsilon_{33}
\end{array}\right],
$$

and the new fundamental cell (lattice vectors) are given by

$$
\bar{\Gamma}=\Gamma+\Delta \Gamma
$$

Substituting the above equation into Eq. (44), we have

$$
\overline{\mathbf{x}}_{j}^{E}=\overline{\boldsymbol{\Gamma}} \cdot \mathbf{x}_{j}^{L}=\mathbf{x}_{j}^{E}+\Delta \boldsymbol{\Gamma} \cdot \mathbf{x}_{j}^{L}
$$

Thus, the strain of the fundamental cell corresponds to non-trivial collective motions of the particle centroids (see Fig. 44). In general, the translational motions of the particles contain the contributions from a random independent part (given by Eq. (4)) and the collective motion imposed by the adaptive fundamental cell. It is this collective motion that enables the algorithm to explore the configuration space more efficiently and to produce highly dense packings.

\section{Checking Overlaps and the Separation Axis Theorem}

Hard polyhedron particles, unlike spheres, ellipsoids and superballs, do not possess simple overlap potential functions. Thus, the check of overlapping for such particles requires other 
techniques. Two convex objects are separated in space if and only if there exist an axis, on which the line segments defined by projections of the two objects do not overlap (see Fig. 5). This statement is usually referred to as the separation axis theorem (SAT) [33].

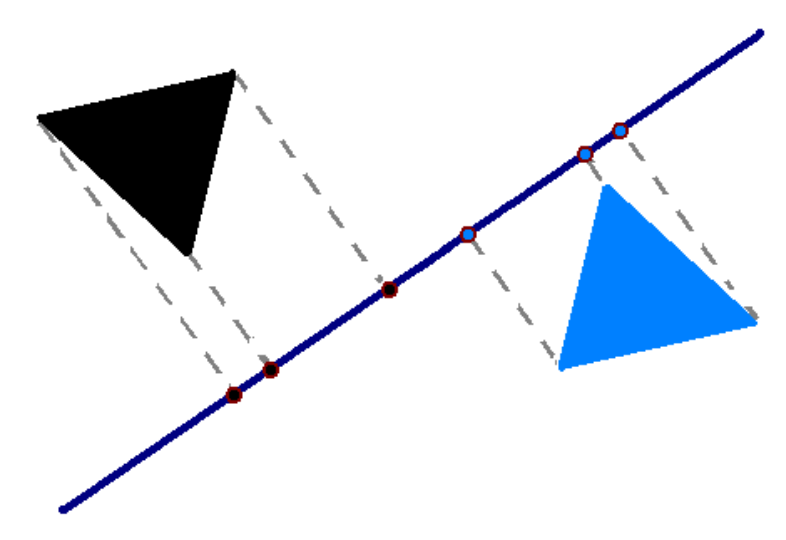

FIG. 5: (color online). Two nonoverlapping particles and one of their separation axes.

For convex polyhedra, the above theorem has a simpler version. Since a polyhedron is completely defined by its vertices, the line segment on the axis is defined by the most separated two projections of the vertices. Moreover, the axis is either perpendicular to one of the faces or perpendicular to a pair of edges, one from each polyhedron. This reduces the number of axes that need to be checked from infinity to $[E(E-1) / 2+2 F]$, where $E$ and $F$ is the number of edges and faces of the polyhedron, respectively. Here we employ the SAT to check for interparticle overlaps numerically up to the highest machine precision.

For polyhedra whose circumscribed and inscribed spheres are well defined, two particles are guaranteed to overlap if the centroidal separation is smaller than the inscribed diameter and guaranteed not to overlap if the centroidal separation is larger than the circumscribed diameter. This pre-check dramatically speeds up the simulations starting from configurations with low densities.

\section{Cell Method and Near-Neighbor List}

The well-developed cell method [25, 26] for particle-system simulation is used here to speed up the process. However, the simulation box (fundamental cell) will not remain a 


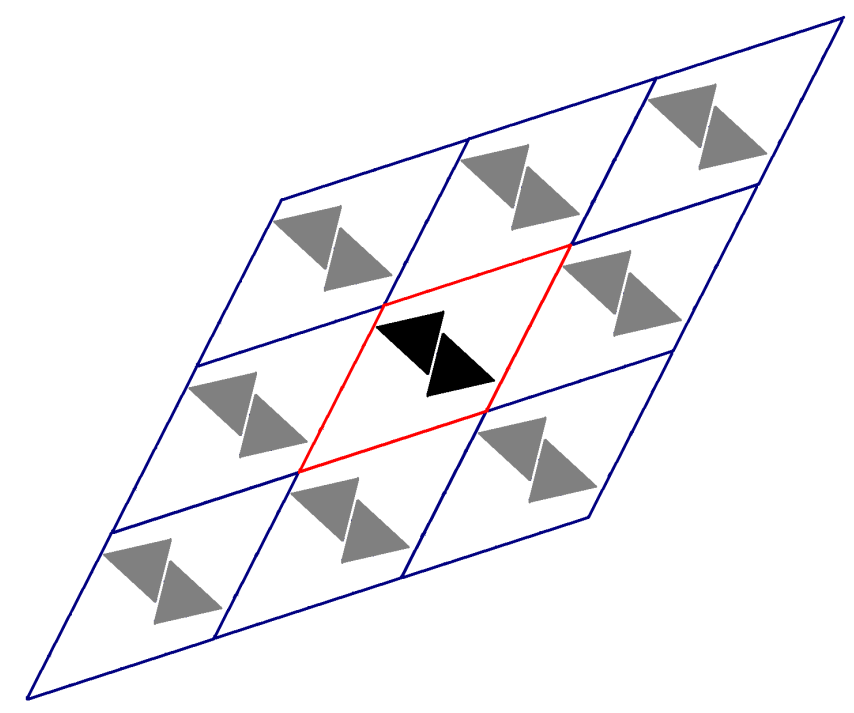

FIG. 6: (color online). The particles (black) in the central fundamental cell (with red boundary) and their images (gray). The distances between the particles in the central cell and all the corresponding images need to be checked.

cube during the simulation and several conventional techniques developed for cubic box need modifications. In particular, to obtain the minimum image distances, all surrounding boxes need to be checked explicitly (see Fig. [6). With the help of the relative-coordinate representation of the particle centroids, the separation vector of a pair of particles is given by

$$
\mathbf{d}_{S}=\boldsymbol{\Gamma} \cdot\left(\mathbf{x}_{i}^{L}-\mathbf{x}_{j}^{L}+\mathbf{L}\right),
$$

where $\mathbf{L}$ is a vector with the values of components to be 1,0 or -1 . Note $\mathbf{L}$ can also be considered as the index of the box that the image particle is in.

The cells are taken to be the same shapes as the simulation box. Partitions of the particle centroids into the cells are also convenient for relative coordinate representations, i.e., the index of the cell (represented by a vector) $\mathbf{C}$ for particle $i$ is given by

$$
\mathbf{C}=\left[N_{C} \mathbf{x}_{i}^{L}\right]
$$

where $[X]$ gives the smallest integer part of $X$ and $N_{C}$ is the number of cells. Note that the boundary deformations and rotations of particles do not affect the cells in which the particles are situated; only translations may cause transitions between the different cells. 
For dense packings, we make use of a near-neighbor list (NNL) to further improve the efficiency of the algorithm [25, 26]. In particular, when the packing density is high, each particle is "trapped" in a "cage" formed by its near neighbors. These near-neighbor configurations are practically stationary, i.e., the particles undergo very small "jiggling" motions. Thus, to check for overlapping between the particles, one only needs to consider a particles nearest neighbors. If a large magnitude translation is made, the NNL needs to be set up again.

\section{APPLICATION OF THE ASC SCHEME TO THE PLATONIC SOLIDS AND RESULTS}

Here we apply the ASC scheme to obtain the densest known packings of the non-tiling Platonic solids. Due to its lack of central symmetry, the tetrahedron presents the greatest challenge for the numerical solution procedure of the ASC scheme because of its tendency to get stuck in local (density) minima, which is a consequence of the associated "rugged" energy or, more precisely, "density" landscape (e.g., the packing density as function of the centroidal positions and orientations of all of the particles in the fundamental cell). Hence, the choice of initial configurations becomes crucial in getting dense tetrahedral packings. By contrast, the central symmetry of the octahedron, dodecahedron and icosahedron results in density landscapes for the numerical procedure that are appreciably less rugged than that for tetrahedral packings.

\section{A. Tetrahedra}

The determination of the densest packings of regular tetrahedra is part of the 18th problem of Hilbert's famous set of problems. It is of interest to note that the densest (Bravais) lattice packing of tetrahedra (which requires all of the tetrahedra to have the same orientations) has the relatively low density $\phi_{\max }^{L}=18 / 49=0.367 \ldots$ and each tetrahedron touches 14 others [34]. Recently, Conway and Torquato showed that the densest packings of regular tetrahedra must not be a Bravais lattice packings, and found packings with density as large as $\phi \approx 0.72$ [13]. One such packing is based upon the filling of "imaginary" icosahedra with the densest arrangement of 20 tetrahedra and then employing the densest lattice packing 
of icosahedra. A slightly higher density was achieved by a perturbation of the so-called "Welsh" packings with density $\phi=17 / 24 \approx 0.708$ [13]. Using "tetrahedral" dice, Chaikin et al. [14] experimentally generated jammed disordered packings of such dice with $\phi \approx 0.75$. Even though the dice are not perfect tetrahedra (because the vertices and edges are slightly rounded), these experimental results suggested that the densest packings of tetrahedra could exceed the highest densities reported by Conway and Torquato. Indeed, Chen [15] has recently discovered a periodic packing of tetrahedra with $\phi=0.7786 \ldots$ [35]. We will call this the "wagon-wheels" packing because the basic subunits consist of two orthogonally intersecting "wagon" wheels. A "wagon wheel" consists of five contacting tetrahedra packed around a common edge (see Fig. 1a of Ref. 13).

TABLE I: Some packing characteristics of certain known tetrahedral packings. Here $\phi$ is the packing density, $N$ is the number of particles in the fundamental cell, and $\bar{Z}$ is the average contact number per particle.

\begin{tabular}{ccccc}
\hline \hline Name & $\phi$ & Locally Jammed & $N$ & $\bar{Z}$ \\
\hline Optimal Lattice [34] & $18 / 49 \approx 0.367346$ & Yes & 1 & 14 \\
Uniform [13] & $2 / 3 \approx 0.666666$ & Yes & 2 & 10 \\
Welsh [13] & $17 / 24 \approx 0.708333$ & No & 34 & 25.9 \\
Icosahedral [13] & 0.716559 & No & 20 & 20.6 \\
Wagon Wheels [15] & 0.778615 & Yes & 18 & 7.1 \\
\hline \hline
\end{tabular}

\section{Initial Conditions}

Because we will use all of the aforementioned tetrahedral packing arrangements as initial conditions in our numerical solution procedure for the ASC optimization problem, we describe them now in a bit more detail. Table 1 summarizes some of their packing characteristics, including the packing density $\phi$, the number of particles $N$ in the fundamental cell, the average number of contacting particles per particle $\bar{Z}$, and whether the packing is locally jammed. A packing is locally jammed if each particle is locally trapped by its neighbors, i.e., it cannot be translated or rotated while fixing the positions and orientations of all the other particles [32, 36]. 
In the optimal lattice packing, each tetrahedron touches 14 others via edges and vertices. In the uniform packing, the tetrahedra are "locked" in a planer layer, and each particle has eight in-plane partial face-to-face contacts and two edge-to-edge contacts (contributed by the two layers above and below), and therefore each particle contacts 10 others. In the icosahedral packing, the tetrahedra have a greater degree of freedom to move, and indeed it is not locally jammed. The 20 tetrahedra inside the imaginary icosahedron are required to meet at its centroid, and so each tetrahedron has 20 vertex-to-vertex contacts. Placing the imaginary icosahedra on the sites of its optimal lattice results in a tetrahedral packing with 12 partial face-to-face contacts. Thus, there are 8 tetrahedra that have 20 contacts per particle and 12 tetrahedra with 21 contacts per particle, and therefore $\bar{Z}=(8 \times 20+12 \times 21) / 20=$ 20.6 .

The Welsh packing is based on the "primitive Welsh" tessellation of $\mathbb{R}^{3}$ into truncated large tetrahedra and small regular tetrahedra [13]. Interestingly, the "primitive Welsh" configuration is closely related to the uniform packing, i.e., it can be constructed by replicating a periodic packing with two truncated large tetrahedra and two small regular tetrahedra in the fundamental cell. Each truncated large tetrahedron can be divided into 12 "Welsh lows", and 4 "Welsh medials," following the notation in Ref. 13. The Welsh medials and Welsh lows are tetrahedra with lower symmetry than regular tetrahedra, which are referred to as the "Welsh highs." To construct the Welsh packing, we insert Welsh highs (regular tetrahedra) into the Welsh low and medial regions. We will refer to the regular tetrahedra that are placed in the Welsh low, medial and high regions as lows, medials and highs, respectively. In the Welsh packing, each high makes 4 face-to-face contacts with 4 medials. Each medial contacts 24 lows and 3 other medials at the centroid of the large truncated tetrahedron; moreover, each medial makes a face-to-face contact with one high. Thus, each medial has 28 contacts. Each low contacts 23 other lows and 4 medials at the centroid of the large truncated tetrahedron. Thus, in total each low has 27 contacts. Each truncated large tetrahedron has 12 lows and 4 medials. Thus, the number of basis particles in the fundamental cell $N=34$ and $\bar{Z}=(12 \times 27+4 \times 28+1 \times 4) / 17=440 / 17=25.882352 \ldots$.

In the fundamental cell of the wagon-wheels packing, there are $N=18$ particles, forming two clusters, each of which includes two "wheels" entangled orthogonally to each other. Each cluster has a central connection particle, an "upper" wheel and a "lower" wheel. The central particle contacts the other 8 tetrahedra through two of its edges. Each of 
the other 8 tetrahedra has 4 edge contacts with particles in its own cluster and 3 partial face-to-face contacts with particles from other clusters. Thus the average contact number $\bar{Z}=(1 \times 8+8 \times 7) / 9=64 / 9=7.111111 \ldots$ The coordinates of all of the 18 tetrahedra in the fundamental cell can be found on the authors' website [37].

\section{Dense Packings}

We employ our algorithm to obtain dense packings of tetrahedra using initial configurations that are based upon the known packings given in Table I as well as certain dilute packings with carefully chosen fundamental cells and the number of particles. Note that these dilute packings were not used as initial configurations to obtain the results reported

in Ref. [27]. A range of initial densities is used to yield the largest possible densities in the final states, as summarized in Table II.

For initial conditions using the known packings in Table प, the fundamental cell is isotropically expanded with the relative coordinates of the tetrahedra fixed so that the initial packing is locally unjammed with a lower density $\phi_{\text {int }}$. The highest density of 0.782 obtained from this subset of initial conditions started from the "wagon-wheels" packing, which was the value reported in Ref. [27].

For the aforementioned dilute packings, a variety of fundamental cell shapes spanning from that for the simple cubic lattice to that of the hexagonal close packing as well as a wide range of particle numbers spanning from 70 to 350 are explored to generate the dense packings. Specifically, the densest known tetrahedral packing is obtained from a dilute initial packing with a rhombical fundamental cell similar to that of the hexagonal close packing and 314 particles (see Table II). The particles are originally placed in the fundamental cell randomly and then the system is sufficiently equilibrated with the fundamental cell fixed. The final packing configuration has a density of about 0.823 and is shown in Fig. 7 from two different viewpoints. The optimized lattice vectors for the densest packing and other characteristics are given in Appendix B. We see that the packing lacks long-range order and is composed of "clusters" of distorted wagon wheels and individual tetrahedra. Thus, we call this dense arrangement the "disordered wagon wheels" packing. We will comment further about the significance of achieving this remarkably high density with a disordered packing in the Discussion and Conclusions (Sec. V). 
TABLE II: Dense tetrahedral packings generated from our algorithm using initial configurations based upon the packings given Table $\$ and the dilute packing as described in the text. Here $\phi_{\text {int }}$ is the initial packing density, $\phi$ is the final packing density, $N$ is the number of tetrahedra in the fundamental cell [38], and $\bar{Z}$ is the average contact number per particle.

\begin{tabular}{cccccc}
\hline \hline Initial Packing & $\phi_{\text {int }}$ & $\phi$ & Locally Jammed & $N$ & $\bar{Z}$ \\
\hline Optimal Lattice & $0.25-0.3$ & 0.695407 & Yes & 27 & 6.6 \\
Uniform & $0.45-0.55$ & 0.665384 & Yes & 54 & 9.8 \\
Welsh & $0.45-0.6$ & 0.752092 & Yes & 34 & 7.4 \\
Icosahedral & $0.45-0.6$ & 0.744532 & Yes & 20 & 7.1 \\
Wagon Wheels & $0.55-0.65$ & 0.782021 & Yes & 72 & 7.6 \\
Disordered Wagon Wheels & $0.005-0.01$ & 0.822637 & Yes & 314 & 7.4 \\
\hline \hline
\end{tabular}

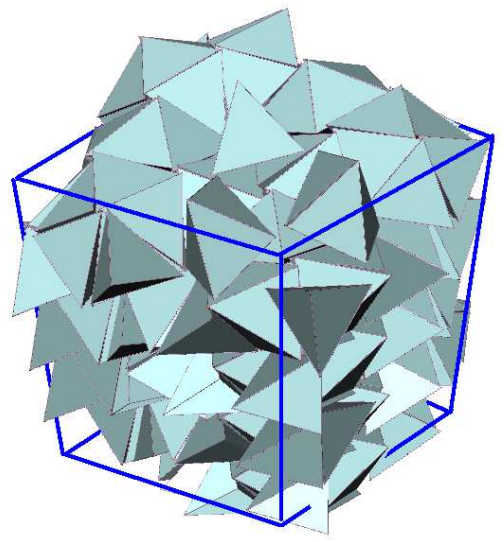

(a)

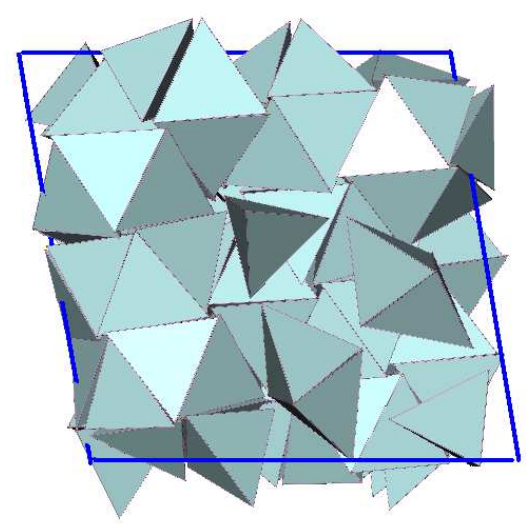

(b)

FIG. 7: The densest known packing of tetrahedra with 314 particles in the fundamental cell. We call this the "disordered wagon-wheels" packing. (a) Viewed from the side. (b) Viewed from the top. It is apparent that the packing lacks long-range order and consists of "clusters" of distorted wagon wheels.

\section{Statistics of the Densest Tetrahedral Packing}

Contact Numbers: The contact numbers of the densest tetrahedral packing as well as those obtained using the other five initial configurations (Table I) are given in Table II, To determine contacting neighbors, it is crucial to find the interparticle gaps first. In particular, 
each of the equilateral triangular faces of the tetrahedra is discretized into $M$ equal-sized smaller triangles and the distances between the vertices of the small triangles of the neighboring tetrahedra are computed, the minimum of which is used as the gap value between the two corresponding tetrahedra. For our packings, when $M>200$, the values of interparticle gaps obtained by this approximation scheme become stable and converge to the true gap values. Like any packing generated via computer simulations, the particles never form perfect contacts and hence contacting neighbors must be determined by setting a tolerance $T$ for the interparticle gaps. Here we choose $T$ to be equal to the mean value of the gaps (of order $10^{-2}$ to $10^{-3}$ of the edge length) such that any gap less than $T$ is associated with a contact. This procedure yields the contact numbers given in Table II In particular, we find that in the disordered wagon wheels packing, there are approximately 6.3 face-to-face or partial face-to-face contacts and 1.1 edge-to-edge contacts.

Face-Normal Correlation Function: An important statistical descriptor for packings of non-spherical particles is the particle orientation correlation function, which measures the extent to which a particle's orientation affects the orientation of another particle at a different position. For highly symmetric particle shapes, it is reasonable to focus on those pair configurations in which the particles are in the same orientation, since particle alignment is associated with dense configurations and phase transitions that may occur. However, because tetrahedra lack central symmetry, we found that face-to-face contacts are favored by the dense packings, instead of face-to-vertex contacts, which are necessarily associated with aligning tetrahedral configurations, such as in the case of the optimal lattice packing.

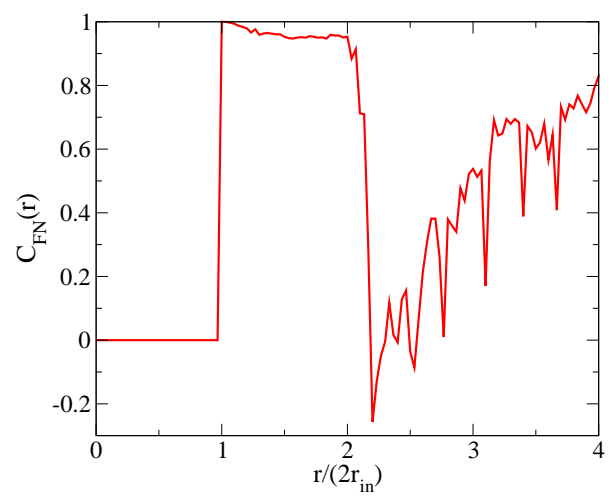

FIG. 8: The face-normal correlation function $C_{F N}(r)$ of the densest known packing of tetrahedra obtained here, where $r_{i n}$ is the radius of the insphere of a tetrahedron. 
Thus, because our interest is in dense tetrahedral packings, we determine the "facenormal" correlation function $C_{F N}(r)$, which we define as the average of the largest negative value of the inner product of two face normals of a pair of tetrahedra separated by a distance $r$. This quantity is plotted in Fig. 8. The strong positive peak values, ranging from $r=$ $2 r_{i n}=d_{0} / \sqrt{6}$ (where $r_{i n}$ is the radius of the insphere of the tetrahedron and $d_{0}$ is the edge length of the particle) to $r$ slightly greater than $2 r_{i n}$, indicate a large number of faceto-face contacts between neighboring particles, implying strong short-range orientational correlations. The fact that $C_{F N}(r)$ plateaus to its maximum or near maximum value, for

the distance interval $r /\left(2 r_{i n}\right) \in(1,2.2)$, indicates that there are partial face-to-face contacts in which particles slide relative to one another such that the distance between the centroids can increase while the face-normal inner product remains the same. The "valleys" of $C_{F N}(r)$ with relatively small magnitudes are manifestations of weak particle alignments (alignment in the same direction), which are local configurations that are necessary "costs" to achieve the largest face-to-face contact numbers on average.

Centroidal Radial Distribution Function: Another statistical descriptor of the structure is the centroidal radial distribution function $g_{2}(r)$. In particular, $g_{2}(r) r^{2} d r$ is proportional to the conditional probability that a particle centroid is found in a spherical shell with thickness $d r$ at a radial distance $r$ from another particle centroid at the origin. It is well established that when there is no long-range order in the system, $g_{2}$ decays to unity very fast. Figure 9 shows the centroidal radial distribution of the densest known tetrahedral packing. We see that $g_{2}(r)$ decays to unity after a few oscillations, indicating that the packing lacks long-range order.

\section{B. Octahedra}

To obtain dense packings of octahedra, we use a wide range of initial configurations. This includes unsaturated packings in which the particles are randomly oriented and positioned with densities spanning the range from 0.2 to 0.35 as well as unsaturated simple cubic (SC) and face-centered cubic (FCC) lattice packings with densities from 0.1 to 0.125 , and the optimal lattice packings with densities from 0.3 to 0.55 are used. We found that, using small compression rates for the random initial configurations and moderate compression rates for the various lattice configurations along with a sufficient number of particle displacements 


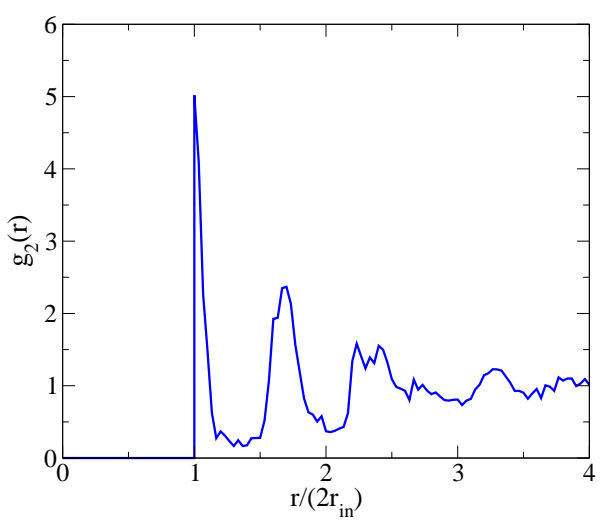

FIG. 9: The centroidal radial distribution function $g_{2}(r)$ of the densest known packing of tetrahedra, where $r_{i n}$ is the radius of the insphere of a tetrahedron.

and rotations, resulted in final configurations with densities larger than 0.93 that are very close in structure and density to the optimal lattice packing. More specifically, starting from the unsaturated SC lattice (with density 0.1 ) and optimal lattice (with density 0.55 ) packings, final packings with densities very slightly larger than the value 0.947003 ... can be obtained, which are extremely close in structure and density to the optimal lattice packing $\left(\phi_{\max }^{L}=0.947368 \ldots\right)$ [22]. A previous study involving an event-driven molecular dynamics growth algorithm for the case of octahedral-like superballs led to the same the densest lattice packing, which lends further credence to the fact that this lattice is indeed optimal among all packings [17]. Each octahedron in the optimal lattice packing, depicted in Fig. 10, contacts 14 others and its lattice vectors are given in Appendix A.

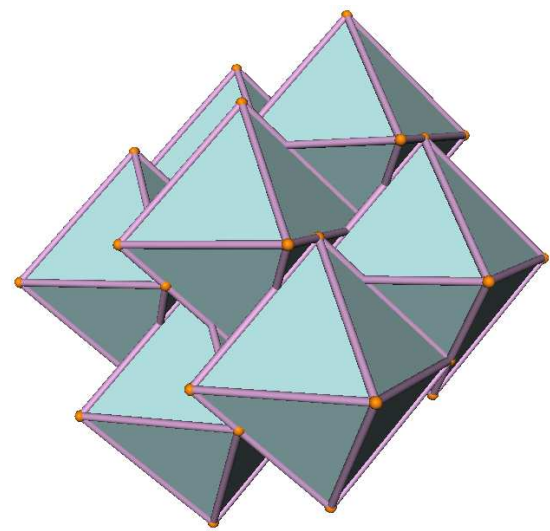

FIG. 10: A portion of the optimal lattice packing of octahedra. 


\section{Icosahedra}

As in the case of octahedra, we use a variety of initial conditions to get dense packings of icosahedra. This includes unsaturated packings in which the icosahedra are randomly oriented and positioned with densities spanning the range from 0.2 to 0.3 as well as various unsaturated lattice packings, such as the BCC, FCC and the densest lattice packing, with densities spanning the range from 0.3 to 0.65 . We found that by starting from a low-density configuration, with a small enough compression rate and sufficient number of particle moves, one can always obtain a final configuration that is very close to the optimal lattice packing with a density $\phi \approx 0.83$. An initial configuration of an unsaturated optimal lattice packing configuration with a density 0.65 gives a final packing with density $0.836315 \ldots$, which is extremely close in structure and density to the optimal lattice packing $\left(\phi_{\max }^{L}=0.836357 \ldots\right)$ [23]. Each icosahedron of the optimal lattice packing, depicted in Fig. 11, contacts 12 others and its lattice vectors are given in Appendix A.

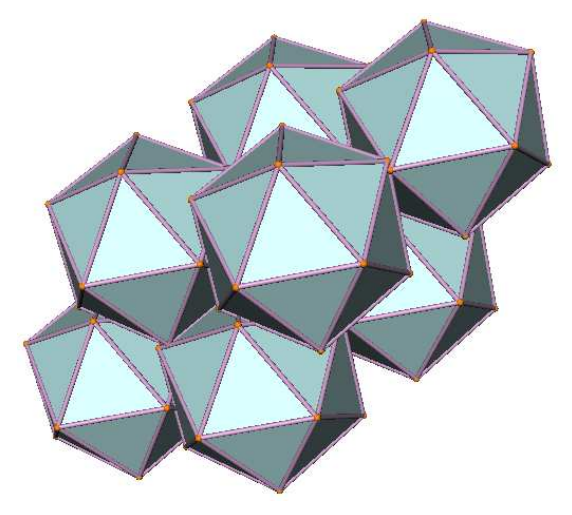

FIG. 11: A portion of the optimal lattice packing of icosahedra.

\section{Dodecahedra}

Unsaturated random packings with densities ranging from 0.15 to 0.3 as well as unsaturated simple cubic and the optimal lattice packings with densities spanning the range from 0.3 to 0.6 are employed as initial configurations to generate dense packings of dodecahedra. We found that it is algorithmically more difficult to avoid local density maxima for dodecahedra than octahedron and icosahedron packings discussed above. For example, starting from 
random configurations, even with sufficiently small compression rate and a large number of particle moves, we can only achieve apparently jammed final packings with densities in the range from 0.83 to 0.85 . When we use an unsaturated optimal lattice packing with density 0.72 as an initial condition, we can generate a final packing with $\phi=0.904002 \ldots$, which is relatively close in structure and density to the optimal lattice packing $\left(\phi_{\max }^{L}=0.904508 \ldots\right)$ [23]. Each dodecahedron of the optimal lattice packing, depicted in Fig. 12, contacts 12 others and its lattice vectors are given in Appendix A.

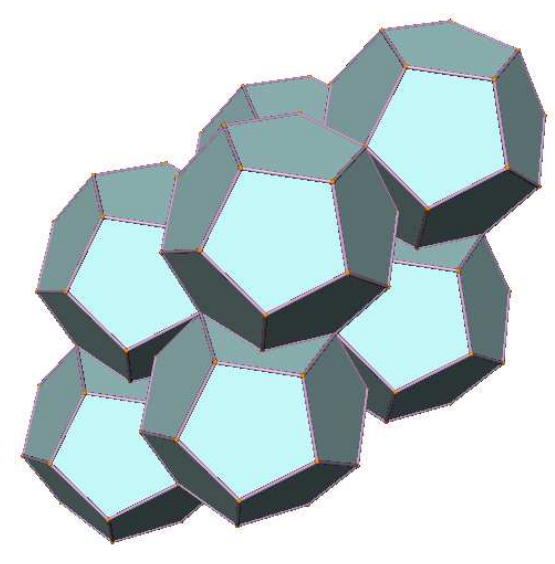

FIG. 12: A portion of the optimal lattice packing of dodecahedra.

We note that the tendency for dodecahedral packings to get stuck in local-density maxima in our algorithm is due to the fact that in some sense the dodecahedron is a shape that is intermediate between the octahedron and icosahedron. Specifically, for octahedral packings, the octahedral symmetry of the particles facilitates the formation of nematic phases at relatively high densities, which can then be easily compressed into a dense crystalline phase with adaptive fundamental cells. An icosahedron is highly isotropic and possesses a large number of faces. Thus, at low densities, the icosahderal packing behaves like a hard-sphere fluid, and it is only near the jamming point that the asphericity of the icosahedron begins to play an important role. Again, by allowing the fundamental cell to adapt in the case of icosahedra, the optimal lattice that best accommodates the packing can be easily identified. However, for our algorithm, the dodecahedral packing behaves differently from either the octahedral or icosahedral packings. In particular, while dodecahedra are more isotropic than octahedra, which strongly suppresses the formation of nematic phases, they have fewer but larger faces than icosahedra, which favors the formation of face-to-face contacts that 
ends up jamming the dodecahedral packing at lower densities than either the octahedral or icosahedral packings.

\section{UPPER BOUND ON THE MAXIMAL DENSITY OF PACKINGS OF NON- SPHERICAL PARTICLES}

Here we derive a simple upper bound on the maximal density $\phi_{\max }$ of a packing of congruent nonspherical particles of volume $v_{p}$ in any Euclidean space dimension $d$. We will see that this bound will aid in our analysis of the optimality of not only the Platonic and Archimedean solids but also superballs. Let $\phi_{\max }^{S}$ be the maximal density of a $d$-dimensional packing of congruent spheres and let $v_{s}$ represent the volume of the largest sphere than can be inscribed in the nonspherical particle.

Lemma: The maximal density of a packing of congruent nonspherical particles is bounded from above according to the following bound [39]:

$$
\phi_{\max } \leq \min \left[\frac{v_{p}}{v_{s}} \phi_{\max }^{S}, 1\right],
$$

where $\min [x, y]$ denotes the minimum of $x$ and $y$.

The proof is straightforward. The maximal packing density $\phi_{\max }$ can be expressed in terms of the maximal number density $\rho_{\max }$ via the relation

$$
\phi_{\max }=\rho_{\max } v_{p}
$$

If we inscribe within each nonspherical particle of the packing the largest possible sphere, it is clear that

$$
\rho_{\max } v_{s} \leq \phi_{\max }^{S}
$$

and therefore combination of the last two equations yields the upper bound of the Lemma. Remark: The upper bound (14) will yield a reasonably tight bound for packings of nonspherical particles provided that the asphericity $\gamma$ of the particle is not large. Here we define the asphericity as the following ratio:

$$
\gamma=\frac{r_{\text {out }}}{r_{\text {in }}}
$$

where $r_{\text {out }}$ and $r_{\text {in }}$ are the circumradius and inradius of the circumsphere and insphere of the nonspherical particle. The circumsphere is the smallest sphere containing the particle. The 
insphere is the largest sphere than can be inscribed in the particle. For a sphere, clearly the asphericity $\gamma=1$. Since upper bound (14) cannot be sharp (i.e., exact) for a nonspherical particle, any packing construction for a nonspherical particle whose density is close to the upper bound (14) is nearly optimal, if not optimal.

In the three-dimensional case, the upper bound (14) becomes

$$
\phi_{\max } \leq \phi_{\max }^{U}=\min \left[\frac{v_{p}}{v_{s}} \frac{\pi}{\sqrt{18}}, 1\right]
$$

We now apply this upper bound to packings of the Platonic and Archimedean solids and compare the bounds to the densities of the corresponding densest lattice packings. Moreover, we apply the upper bounds to superball and ellipsoid packings.

\section{A. Platonic Solids}

Table \II compares the density of the densest lattice packings of the Platonic solids to the corresponding upper bounds on the maximal density for such packings. The large asphericity and lack of central symmetry of the tetrahedron is consistent with the large gap between the upper bound density and densest lattice packing density, and the fact that there are nonlattice packings with density appreciably greater than $\phi_{\max }^{L}$. On the other hand, the central symmetry of the octahedron, dodecahedron and icosahedron and their associated relatively small asphericities explain the corresponding small differences between $\phi_{\max }^{L}$ and $\phi_{\max }^{U}$ and is consistent with our simulation findings that indicate that their optimal arrangements are their respective densest lattice packings.

\section{B. Archimedean Solids}

We also compute the upper bound (18) for each of the 13 Archimedean solids and compare them to the densities of the corresponding densest lattice packings [23, 34]. (In Appendix A we provide the lattice vectors for the optimal lattice packings of the Archimedean solids). Table IV summarizes the upper bounds on the maximal density for such packings. Not surprisingly, the truncated tetrahedron (the only Archimedean solid that is not centrally symmetric) has a large asphericity, implying that there are denser non-lattice packings, as we explicitly identify in Section $\mathrm{V}$. The central symmetry of the majority of the Platonic 
TABLE III: Comparison of the densities of the densest lattice packings for the Platonic solids [22, 23, 34] to the corresponding upper-bound densities as obtained from (18). Here $v_{p}$ is volume of the polyhedron with unit edge length, $r_{\text {in }}$ and $r_{\text {out }}$ are the radii of the insphere and circumsphere of the polyhedron with unit edge length, respectively, $\gamma=r_{\text {out }} / r_{\text {in }}$ is the asphericity, $\phi_{\max }^{L}$ is the density of the optimal lattice packing and $\phi_{\max }^{U}$ is the upper bound (18). The numerical values are reported up to the sixth decimal place. The naming code used here is the same one used in Fig. 1.

\begin{tabular}{ccccccc}
\hline \hline Name & $v_{p}$ & $r_{\text {in }}$ & $r_{\text {out }}$ & $\gamma$ & $\phi_{\max }^{L}$ & $\phi_{\max }^{U}$ \\
\hline Tetrahedron (P1) & $\frac{\sqrt{2}}{12}$ & $\frac{\sqrt{6}}{12}$ & $\frac{\sqrt{6}}{4}$ & 3 & 0.367346 & 1 \\
Icosahedron (P2) & $\frac{5(3+\sqrt{5})}{12}$ & $\frac{3 \sqrt{3}+\sqrt{15}}{12}$ & $\frac{\sqrt{10+2 \sqrt{5}}}{4}$ & 1.258410 & 0.836357 & 0.893417 \\
Dodecahedron (P3) & $\frac{15+7 \sqrt{5}}{4}$ & $\frac{\sqrt{250+110 \sqrt{5}}}{20}$ & $\frac{\sqrt{3}+\sqrt{15}}{4}$ & 1.258410 & 0.904508 & 0.981162 \\
Octahedron (P4) & $\frac{\sqrt{2}}{3}$ & $\frac{\sqrt{6}}{6}$ & $\frac{\sqrt{2}}{2}$ & 1.732050 & 0.947368 & 1 \\
Cube (P5) & 1 & $\frac{1}{2}$ & $\frac{\sqrt{3}}{2}$ & 1.732050 & 1 & 1 \\
\hline \hline
\end{tabular}

and Archimedean solids and their associated relatively small asphericities explain the corresponding small differences between $\phi_{\max }^{L}$ and $\phi_{\max }^{U}$ and is consistent with our simulation findings that strongly indicate that their optimal arrangements are their respective densest lattice packings.

\section{Superballs}

The upper bound (18) in the case of superballs [17] can be expressed analytically for all values of the deformation parameter $p$. A three-dimensional superball is a centrally symmetric body in $\mathbb{R}^{3}$ occupying the region

$$
\left|x_{1}\right|^{2 p}+\left|x_{2}\right|^{2 p}+\left|x_{3}\right|^{2 p} \leq 1
$$

where $x_{i}(i=1,2,3)$ are Cartesian coordinates and $p \geq 0$ is the deformation parameter, which indicates to what extent the particle shape has deformed from that of a sphere $(p=1)$. A superball can possess two types of shape anisotropy: cubic-like shapes for $1 \leq p \leq \infty$, with $p=\infty$ corresponding to the perfect cube, and octahedral-like shapes for $0 \leq p \leq 1$, with $p=1 / 2$ corresponding to the perfect regular octahedron. In Ref. 17, event-driven molecular dynamics growth algorithms as well as theoretical arguments led to the conjecture that 
TABLE IV: Comparison of the densities of the densest lattice packings for the Archimedean solids to the corresponding upper-bound densities as obtained from (18). Except for the cubeoctahedron [34], the densities of the densest lattice packings were obtained by Betke and Henk [23]. Here $v_{p}$ is volume of the polyhedron with unit edge length, $r_{\text {in }}$ and $r_{\text {out }}$ are the radii of the insphere and circumsphere of the polyhedron with unit edge length, respectively, $\gamma=r_{\text {out }} / r_{\text {in }}$ is the asphericity, $\phi_{\max }^{L}$ is the density of the optimal lattice packing, $\phi_{\max }^{U}$ is the upper bound (18) and $t=[1+$ $\left.(19-3 \sqrt{33})^{\frac{1}{3}}+(19+3 \sqrt{33})^{\frac{1}{3}}\right] / 3 \approx 1.83929 \ldots$ is the Tribonacci constant. The numerical values are reported up to the sixth decimal place. The naming code used here is the same one used in Fig. 3.

\begin{tabular}{|c|c|c|c|c|c|c|}
\hline Name & $v_{p}$ & $r_{i n}$ & $r_{\text {out }}$ & $\gamma$ & $\phi_{\max }^{L}$ & $\phi_{\max }^{U}$ \\
\hline Truncated Tetrahedron (A1) & $\frac{23 \sqrt{2}}{12}$ & $\frac{\sqrt{6}}{4}$ & $\frac{\sqrt{22}}{4}$ & 1.914854 & 0.680921 & 1 \\
\hline Truncated Icosahedron (A2) & $\frac{125+43 \sqrt{5}}{4}$ & $\frac{\sqrt{21+9 \sqrt{5}}}{2 \sqrt{2}}$ & $\frac{\sqrt{58+18 \sqrt{5}}}{4}$ & 1.092945 & 0.784987 & 0.838563 \\
\hline Snub Cube (A3) & $\frac{3 \sqrt{t-1}+4 \sqrt{t+1}}{3 \sqrt{2-t}}$ & $\sqrt{\frac{t-1}{4(2-t)}}$ & $\sqrt{\frac{3-t}{4(2-t)}}$ & 1.175999 & 0.787699 & 0.934921 \\
\hline Snub Dodecahedron (A4) & 37.616654 & 1.980915 & 2.155837 & 1.088303 & 0.788640 & 0.855474 \\
\hline Rhombicicosidodecahedron (A5) & $95+50 \sqrt{5}$ & 3.523154 & $\frac{\sqrt{31+12 \sqrt{5}}}{2}$ & 1.079258 & 0.804708 & 0.835964 \\
\hline Truncated Icosidodecahedron (A6) & $\frac{60+29 \sqrt{5}}{3}$ & 2.016403 & $\frac{\sqrt{11+4 \sqrt{5}}}{2}$ & 1.107392 & 0.827213 & 0.897316 \\
\hline Truncated Cuboctahedron (A7) & $\frac{12+10 \sqrt{2}}{3}$ & $\frac{1+\sqrt{2}}{2}$ & $\frac{\sqrt{5+2 \sqrt{2}}}{2}$ & 1.158941 & 0.849373 & 0.875805 \\
\hline Icosidodecahedron (A8) & $\frac{45+17 \sqrt{5}}{6}$ & $\sqrt{\frac{5+2 \sqrt{5}}{5}}$ & $\frac{1+\sqrt{5}}{2}$ & 1.175570 & 0.864720 & 0.938002 \\
\hline Rhombicuboctahedron (A9) & $22+14 \sqrt{2}$ & $\frac{1+2 \sqrt{2}}{2}$ & $\frac{\sqrt{13+6 \sqrt{2}}}{2}$ & 1.210737 & 0.875805 & 1 \\
\hline Truncated Dodecahedron (A10) & $\frac{5(99+47 \sqrt{5})}{12}$ & $\frac{\sqrt{25+11 \sqrt{5}}}{2 \sqrt{2}}$ & $\frac{\sqrt{74+30 \sqrt{5}}}{4}$ & 1.192598 & 0.897787 & 0.973871 \\
\hline Cuboctahedron (A11) & $\frac{5 \sqrt{2}}{3}$ & $\frac{\sqrt{2}}{2}$ & 1 & 1.414213 & 0.918367 & 1 \\
\hline Truncated Cube (A12) & $\frac{21+14 \sqrt{2}}{3}$ & $\frac{1+\sqrt{2}}{2}$ & $\frac{\sqrt{7+4 \sqrt{2}}}{2}$ & 1.473625 & 0.973747 & 1 \\
\hline Truncated Octahedron (A13) & $8 \sqrt{2}$ & $\frac{\sqrt{6}}{2}$ & $\frac{\sqrt{10}}{2}$ & 1.290994 & 1 & 1 \\
\hline
\end{tabular}

the densest packings of superballs for all convex shapes $(1 / 2 \leq p \leq \infty)$ are certain lattice packings, depending on the value of the deformation parameter $p$.

For convex superballs in the octahedral regime $(0.5<p<1)$, the upper bound (18) is explicitly given by

$$
\phi_{\max }^{U}=\frac{\sqrt{6}}{108 p^{2}} \times 3^{\frac{1}{2 p}} B\left(\frac{1}{2 p}, \frac{2 p+1}{2 p}\right) B\left(\frac{1}{2 p}, \frac{p+1}{p}\right)
$$


similarly, for superballs in the cubic regime $(p>1)$, the upper bound (18) is given by

$$
\phi_{\max }^{U}=\frac{\sqrt{2}}{4 p^{2}} B\left(\frac{1}{2 p}, \frac{2 p+1}{2 p}\right) B\left(\frac{1}{2 p}, \frac{p+1}{p}\right),
$$

where $B(x, y)=\Gamma(x) \Gamma(y) / \Gamma(x+y)$ and $\Gamma(x)$ is the Euler Gamma function. Here and in the subsequent paragraphs concerning ellipsoids, we only state the nontrivial part of the upper bound (18). For $p$ near the sphere point $(p=1)$, the densest lattice packings [17] have densities that lie relatively close to the corresponding upper-bound values. For example, for $p=0.99,0.98$ and $0.97, \phi_{\max }^{U}=0.745327 \ldots, 0.750274 \ldots$, and $0.755325 \ldots$, respectively, which is to be compared to $\phi_{\max }^{L}=0.740835 \ldots, 0.741318 \ldots$, and $0.741940 \ldots$, respectively. For $p=1.01,1.02$ and $1.03, \phi_{\max }^{U}=0.747834 \ldots, 0.755084 \ldots$, and $0.762233 \ldots$, respectively, which is to be compared to $\phi_{\max }^{L}=0.741720 \ldots, 0.742966 \ldots$, and $0.744218 \ldots$, respectively.

The fact that the constructed densest lattice packings of superballs have density $\phi_{\max }^{L}$ relatively close to the upper bound value $\phi_{\max }^{U}$ strengthens the arguments made in Ref. 17 that suggested that the optimal packings are in fact given by these arrangements. If the optimal packings around $p=1$ are indeed the lattice packings, it would be surprising that for other values of $p$ a continuous deformation of the superballs would result in a transition from lattice packings to denser non-lattice packings.

\section{Ellipsoids}

In the case of ellipsoids in which the the ratio of the three semiaxes are given by $1: \alpha: \beta$ $(\alpha, \beta \geq 1)$, the upper bound (18) becomes

$$
\phi_{\max }^{U}=\alpha \beta \frac{\pi}{\sqrt{18}}
$$

For prolate spheroids $\beta=1$, and the bound becomes

$$
\phi_{\max }^{U}=\alpha \frac{\pi}{\sqrt{18}}
$$

For oblate spheroids with $\alpha=\beta>1$, the bound is given by

$$
\phi_{\max }^{U}=\alpha^{2} \frac{\pi}{\sqrt{18}}
$$

The upper bounds for ellipsoids generally do not work as well as those for the centrally symmetric Platonic and Archimedean solids, and superballs. This is due to the fact that the 
three principle directions (axes) are not equivalent for an ellipsoid, i.e., it generally possesses different semiaxes. Note that the three equivalent principle (orthogonal) axes (directions) of a centrally symmetric particle are those directions that are two-fold rotational symmetry axes such that the distances from the particle centroids to the particle surfaces are equal. In addition, the asphericity of an ellipsoid increases linearly as the largest aspect ratio $\alpha$ increases without limit. By contrast, the three principle directions are equivalent for the centrally-symmetric Platonic and Archimedean solids as well as for superballs, for which the asphericity is always bounded and close to unity (see Tables III and IV]). Thus, we see that an asphericity value close to unity is a necessary condition to have a centrally symmetric particle in which the three principle directions are equivalent.

\section{DISCUSSION AND CONCLUSIONS}

We have formulated the problem of generating dense packings of nonoverlapping polyhedra within an adaptive fundamental cell subject to periodic boundary conditions as an optimization problem, which we call the Adaptive Shrinking Cell (ASC) scheme. The procedure allows both a sequential search of the configurational space of the particles and the space of lattices via an adaptive fundamental cell that shrinks on average to obtain dense packings. We have applied the ASC to generate the densest known packings of the Platonic solids.

For tetrahedra, we find a packing with density $\phi \approx 0.823$, which is a periodic (nonBravais lattice) packing with a complex basis. Unlike the other Platonic solids, finding dense packings of tetrahedra with our algorithm requires having good initial configurations. The densest packing was found using 314 particles in a rhombical fundamental cell that is similar to that of the hexagonal close packing. As we stressed in our earlier work [27] and continue to confirm in this paper, it is possible that denser packings of tetrahedra will involve increasingly larger numbers of particles in the fundamental cell. In fact, the higher density found here is realized by a larger periodic packing (314 particles per cell) than the one reported in Ref. [27] (72 particles per cell) with density 0.782. It is apparent the obtained densest known tetrahedral packing is disordered in the sense that it possesses no long-range order, at least on the scale of the simulation box. This packing can be considered to be a disordered "mixture" of distorted wagon wheels and individual tetrahedra and it 
is distinct from the one reported in Ref. [27] in both the arrangement of the wagon wheels and the extent of the distortion of the wagon wheels. Although we cannot rule out the possibility of the existence of denser packings involving a more ordered arrangement of the wagon wheels and individual tetrahedra, it is reasonable to expect that wagon wheels will be key building blocks in denser packings and should at least be slightly distorted to fill the interparticle gaps in wagon-wheel clusters more efficiently, which necessarily introduces a certain degree of disorder to the packing. If denser packings of tetrahedra must involve larger number of particles without long-range order, than it raises the amazing prospect that the densest packings of tetrahedra might be truly disordered, due to the geometrical frustration associated with the lack of central symmetry of a tetrahedron and that tetrahedra cannot tile space. This would be the first example of a maximally dense packing of congruent convex three-dimensional particles without long-range order. However, we cannot offer definitive conclusions about this possibility at this stage. It is clear that in future work in the search for denser packings, increasingly larger number of tetrahedra must be considered, which can only be studied using greater computational resources.

Our simulation results and rigorous upper bounds strongly suggest that the optimal lattice packings of the centrally symmetric Platonic solids (octahedra, dodecahedra and icosahedra) are indeed the densest packings of these particles, especially since these arise from a variety of initial "dilute" multi-particle configurations within the fundamental cell [40]. It is noteworthy that a different simulation procedure (an event-driven molecular dynamics growth algorithm with an adaptive fundamental cell) has recently been used to demonstrate that the densest packings of octahedral-like superballs (which contains the perfect octahedron) are likely to be the optimal lattice packings [17]. Moreover, the fact that the optimal lattice packing densities of certain centrally symmetric nonspherical particles, such as the Archimedean solids with central symmetry and convex superballs, are relatively close to their upper bounds as well as other theoretical arguments given below suggest that the densest packings of these particles may also be given by their optimal lattice packings.

It is crucial to stress that the nonspherical particles in this family do not deviate appreciably from a sphere, i.e., their corresponding asphericity $\gamma$ is always bounded and relatively close to unity, and, moreover, their three principle axes (directions) are equivalent. This is in contrast to the ellipsoid, which, although centrally symmetric, generally possesses three principle axes (directions) that are inequivalent and its asphericity can increase without limit 
as the largest aspect ratio grows. These characteristics suggest that the densest ellipsoid arrangements are non-lattice packings, which indeed has been verified [12].

Our simulation results and the ensuing theoretical arguments lead to the following conjecture:

Conjecture 1: The densest packings of the centrally symmetric Platonic solids are given by their corresponding optimal lattice packings.

We now sketch what could be the major elements of a proof of this conjecture. In the case of each of the Platonic solids, face-to-face contacts are favored over vertex-to-face contacts to achieve higher packing densities, since the former allows the particle centroids to get closer to one another. Such contacting neighbor configurations around each particle reduce the volume of the corresponding convex hull joining the centroids of the contacting particles, and the fraction of space covered by the particles within this convex hull should be increased; see Fig. 13 for a two-dimensional illustration.

To achieve the densest packing, the fraction of space covered by the particles within the convex hull should ideally be maximized and so should the number of face-to-face contacts per particle. Of course, this is only a local criterion that may not be consistent with the densest global packing. However, it will be seen that the equivalence of the three principle directions of a centrally symmetric Platonic solid is crucial for this local optimization criterion to be consistent with the globally densest packing.

It is noteworthy that orienting each of the particles in the packings of centrally symmetric Platonic solids enable a larger number of face-to-face contacts and thus allows the maximal fraction of space covered by the particles within the convex hull. For example, a particle with $F$ faces, possesses $F / 2$ families of axes that go through the centroid of the particle and intersect the centrally-symmetric face pairs such that the particles (in the same orientation) with their centroids arranged on these axes form face-to-face contacts.

The requirement that the particles have the same orientation is globally consistent with a lattice packing. Indeed, in the optimal lattice packings of the centrally symmetric Platonic solids, each particle has the maximum number of face-to-face contacts that could be obtained without violating the impenetrability condition. It is highly unlikely that such particles possessing three equivalent principle directions and aligned in the same direction could form a more complicated non-lattice periodic packings with densities that are larger than the optimal lattice packings. Such non-lattice packings would arise to take advantage of the 


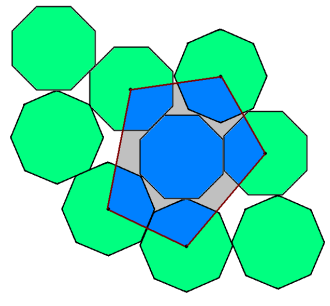

(a)

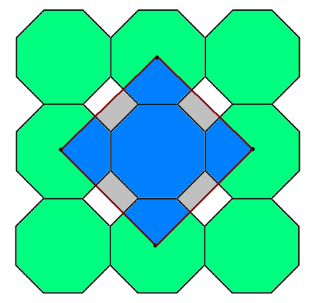

(b)

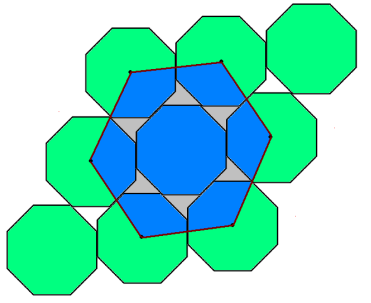

(c)

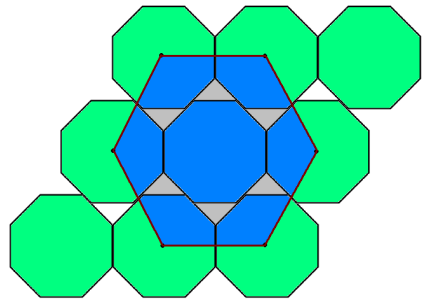

(d)

FIG. 13: (color online). Illustration of our conjecture concerning the optimal packings of centrallysymmetric particles using two-dimensional octagonal packings. It is shown how different particle orientations and arrangements affect the number of face-to-face contacts and the area of the convex hulls joining the centers of the neighboring particles around a central one. (a) The octagons do not have the same orientation and the number of face-to-face contacts is small. (b) The octagons are aligned with 4 contacts per particle to form a lattice packing, but the area of the convex hull associated with the central particle is not minimized. (c) The octagons are aligned with 6 contacts per particle to form a lattice packing, but although the area of the convex hull associated with the central particle is smaller than in (b), it is not minimized. (d) The octagons are aligned with 6 contacts per particle to form a lattice packing, and the area of the convex hull associated with the central particle is minimized. This minimization corresponds to finding the the minimal circumscribing hexagon and therefore corresponds to the optimal lattice packing [41].

rotational degrees of freedom. By requiring particle alignments, only translational degrees of freedom remain and hence optimization over these degrees of freedom would lead to the globally optimal packings, which should be lattice packings. Constraining rotational degrees of freedom in this way in a non-lattice packing would at best lead to a local optimum in density. This conclusion is clearly supported by our simulations, which use multiple-particle configurations in the fundamental cell and only produce the optimal lattice packings.

In the aforementioned arguments, a key step that is difficult to prove is the observation that alignment of each of the particles maximizes the number of face-to-face contacts and thus the fraction of space covered by the particles within the convex hull joining the centroids of the contacting neighbors around a central polyhedron. A rigorous verification of this step would lead to the consistency of the aforementioned local and global optimization criteria. 
It is noteworthy that in two dimensions, our local criterion to achieve the optimal packings of centrally symmetric regular polygons (the two-dimensional counterparts of the centrally symmetric Platonic solids) amounts to the identification of optimal neighbor configurations with the maximal edge-to-edge contacts per particle such that the convex hull joining the centroids of the contacting neighbors is minimal. This statement is rigorously true in two dimensions because it is tantamount to a theorem due to Fejes Tóth [41], which states that the densest packing of a centrally symmetric particle in two dimensions can be obtained by circumscribing the particle with the minimal centrally symmetric hexagon, which of course tiles $\mathbb{R}^{2}$. Finding the minimal circumscribing centrally symmetric hexagon is equivalent to finding the minimal area convex hull joining the centroids of the six contacting particles (the maximal number can be obtained in two dimensions for centrally symmetric particles). The fact that local optimality is consistent with the global optimality in two dimensions (e.g., a centrally symmetric hexagon can always tessellate the space) does not hold in three dimensions. Although we have presented a three-dimensional generalization of the Fejes Tóth theorem, by replacing the minimal hexagon with the minimal convex-hull volume, as pointed out before, it is extremely difficult to provide a rigorous proof for it.

Although Conjecture 1 applies to the centrally symmetric Platonic solids, all of our arguments apply as well to each of the centrally symmetric Archimedean solids. It cannot be true for the truncated tetrahedron, which is the only non-centrally symmetric Archimedean solid. Specifically, it immediately follows from the results of Ref. 13 that a non-lattice packing of truncated tetrahedra can be constructed based on the "primitive Welsh" tessellation (i.e., by removing the small regular tetrahedra) that possesses the density $\phi=23 / 24=0.958333 \ldots$, which is appreciably larger than the optimal-lattice-packing density of $\phi_{\max }^{L}=0.680921 \ldots$, and contains two particle centroids per fundamental cell. In fact, given that the truncated tetrahedra cannot tile space, the density of the "primitive Welsh" packing of truncated tetrahedra is so large that it may be the optimal packing of such particles. The lattice vectors of this periodic packing are given in Appendix C.

Since the arguments used to justify Conjecture 1 apply equally well to the 12 centrally symmetric Archimedean solids, we are led to the following more general conjecture:

Conjecture 2: The densest packings of the centrally symmetric Platonic and Archimedean solids are given by their corresponding optimal lattice packings.

The aforementioned arguments can also be extended to the case of superballs, but here 
the local principle curvatures at the contacting points should be sufficiently small so as to maximize the fraction of space covered by the particles within the convex hull joining the centroids of the neighbors. The central symmetry and equivalence of the three principle directions of superballs means that dense packings of such objects are favored when the particles are aligned, which again leads to consistency between local and global optimality. The fact that the optimal lattice packing densities of superballs is relatively close to the upper-bound values, at least around the sphere point, as well as results from previous molecular dynamics simulations [17] also strongly suggest that the densest packings of these particles are given by their corresponding optimal lattice packings.

It is noteworthy that under the assumption that Conjecture 2 is valid, one has upper bounds that are the complete analog of (14), i.e.,

$$
\phi_{\max } \leq \min \left[\frac{v_{p}}{v_{c}} \phi_{\max }^{C}, 1\right]
$$

where $v_{c}$ is the volume of an appropriately chosen centrally symmetric Platonic or Archimedean solid and $\phi_{\max }^{C}$ is the corresponding optimal density for such a solid, which according to Conjecture 2 is the optimal lattice packing. This bound will generally be sharper than bound (14) because the reference optimal packing is less symmetric than the sphere. For example, the conjectured bound (25) will be sharp for slightly deformed Platonic and Archimedean solids or nonspherical particles derived by smoothing the vertices, edges and faces of polyhedra.

Our work also naturally leads to another conjecture:

Conjecture 3: The optimal packing of any convex, congruent polyhedron without central symmetry generally is not a (Bravais) lattice packing.

In other words, the set of such polyhedra whose optimal packing is not a lattice is overwhelmingly larger than the set whose optimal packing is a lattice. We have seen that because the regular tetrahedron and truncated tetrahedron lack central symmetry, dense packings of such objects favor face-to-face contacts. Such orientations immediately eliminate the possibility that lattice packings (in which particles must have the same orientations) are optimal. Similarly, it is very plausible that dense packings of most convex, congruent polyhedra without central symmetry are facilitated by face-to-face contacts and hence the optimal packings cannot be lattices. For example, consider a square with one missing corner, i.e., an isosceles triangle with a right angle (see Fig. 14). At first glance, one might surmise that if the missing 


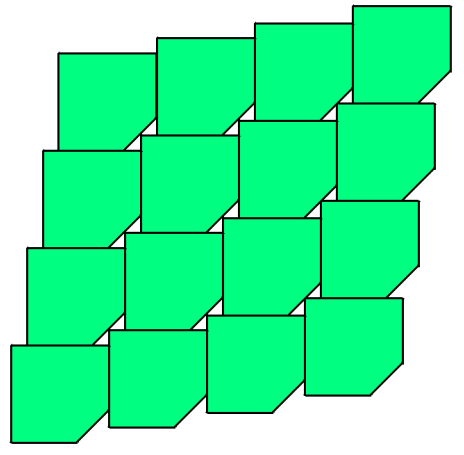

(a)

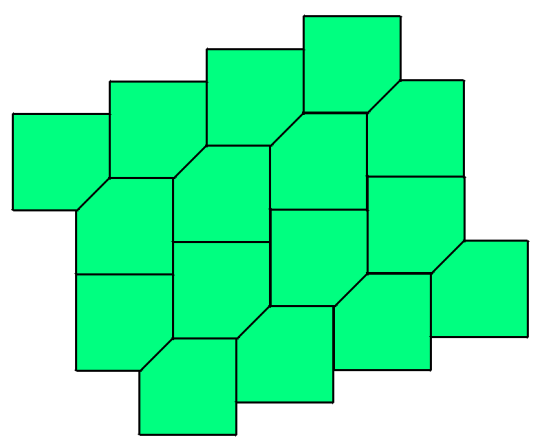

(b)

FIG. 14: (color online). Portions of two packing configurations of pentagons obtained by cutting off a corner (isosceles triangle with a right angle) of a square. (a) The optimal lattice packing and (b) a two-particle basis periodic packing that tiles the plane. Let side length of the square be 1 and the lengths of the equal sides of the isosceles triangle be $\beta \in(0,1)$. The lattice vectors of the optimal lattice packing are $\mathbf{e}_{1}^{L}=\mathbf{i}-\frac{\beta}{2} \mathbf{j}, \mathbf{e}_{2}^{L}=\left(1-\frac{\beta}{2}\right) \mathbf{i}+(1-\beta) \mathbf{j}$ and the lattice vectors of the periodic packing are $\mathbf{e}_{1}^{P}=\mathbf{i}+\beta \mathbf{j}, \mathbf{e}_{2}^{P}=(1-\beta) \mathbf{i}+(2-\beta) \mathbf{j}$ with one particle at origin and the other at $\mathbf{b}_{1}=(1-\beta) \mathbf{i}+(1-\beta) \mathbf{j}$, where $\mathbf{i}, \mathbf{j}$ are the unit vectors along the two orthogonal coordinate directions, coinciding with two orthogonal sides of the square. The density (covering fraction) of the optimal lattice packing is $\phi_{\max }^{L}=\left(1-\frac{\beta^{2}}{2}\right) /\left(1-\frac{\beta^{2}}{4}\right)$ and the density of the periodic packing is $\phi_{\max }^{P}=1$. It can be seen that for all $0<\beta<1, \phi_{\max }^{L}$ is always smaller than $\phi_{\max }^{P}$.

piece is sufficiently small, the original lattice packing should still be optimal or nearly optimal (see Fig. 14a), since lattice packings are optimal for squares. However, no matter how small the missing piece may be, a periodic packing in which the fundamental cell contains two pentagons can be constructed that tile the plane (see Fig. 14b). This is done by taking advantage of the asymmetry of the particle. Thus, we see from this counterintuitive example that if the particle does not possess central symmetry, it is possible to exploit its rotational degrees of freedom to yield a periodic packing with a complex basis that are generally denser than the optimal lattice packing. On the other hand, there are special cases where the lattice will be optimal, such as for the rhombic dodecahedron that has one corner clipped [43]. However, these special cases are overwhelmed in number by those whose optimal packings are not lattices. If Conjecture 3 is valid, it also applies to nonspherical particles derived by 
smoothing the vertices, edges and faces of polyhedra provided that the particle curvature at face-to-face contacts is sufficiently small.

It should not go unnoticed that the densest packings of all of the Platonic and Archimedean solids reported here as well as the densest known packings of superballs [17] and ellipsoids [12] have densities that exceed the optimal sphere packing density $\phi_{\max }^{S}=\pi / \sqrt{18}=0.7408 \ldots$ These results are consistent with a conjecture of Ulam who proposed, without any justification, that the optimal density for packing congruent spheres is smaller than that for any other convex body [42]. The sphere is perfectly isotropic with an asphericity $\gamma$ of unity, and therefore, as noted earlier, its rotational degrees of freedom are irrelevant in affecting its packing characteristics. On the other hand, each of the aforementioned convex non-spherical particles break the continuous rotational symmetry of the sphere and thus its broken symmetry can be exploited to yield the densest possible packings. However, broken rotational symmetry in and of itself may not be sufficient to satisfy Ulam's conjecture if the convex particle has a little or no symmetry.

It will also be interesting to determine whether our conjecture can be extended to other polyhedral packings. The infinite families of prisms and antiprisms [44] provide such a class of packings. A prism is a polyhedron having bases that are parallel, congruent polygons and sides that are parallelograms. An antiprism is a polyhedron having bases that are parallel, congruent polygons and sides that are alternating bands of triangles. Prisms with an even number of sides and antiprisms are centrally symmetric. Although prisms and antiprisms are naturally grouped with the Archimedean solids (i.e., they are polyhedra in which the same regular polygons appear at each vertex), they are generally much less symmetric than either the Platonic or Archimedean solids. Moreover, even the centrally symmetric prisms and antiprisms generally do not possess three equivalent directions. Thus, it is less obvious whether Bravais lattices would still provide the optimal packings for these solids, except for prisms that tile space (e.g., hexagonal prism or rhombical prisms). In future work, it would be desirable to test whether our conjecture extends to prisms and antiprisms that possess central symmetry and three equivalent directions using the ASC scheme.

It is worth noting that in four dimensions, the analogs of the tetrahedron, cube, octahedron, dodecahedron and icosahedron are the four-dimensional regular simplex, hypercube, cross polytope, 120-cell and 600-cell, respectively. All of these four-dimensional polytopes possess central symmetry, except for the simplex [45]. While the hypercube and cross poly- 
tope tile $\mathbb{R}^{4}$, the optimal packings of simplices are still likely to be non-lattices. Since our conjecture for the three-dimensional Platonic solids should still apply in four dimensions, the densest packings of the 120-cell and 600-cell could be their corresponding optimal lattice packings. The cross polytopes for $d \geq 5$ no longer tile space, and their optimal packings may still be their densest lattice packings provided that $d$ is sufficiently small. However, in sufficiently high dimensions, the densest lattice packings of the centrally symmetric polytopes are probably no longer optimal, since lattice packings in high dimensions are known to possess huge "holes" into which additional particles can be inserted, yielding higher packing densities with possibly non-lattice arrangements. Indeed, it has recently been argued that disordered sphere packings in very high dimensions could be denser than any ordered packing [46].

Recent progress in particle synthesis methods have enabled the production of a wide spectrum of nanoparticle shapes such as tetrahedra [47], cubes [48], icosahedra [49] and prisms [50]. In such applications, it would be of great interest to predict the corresponding crystal structures, which might possess unusual symmetries and properties. The idea of incorporating collective particle motions due to the adaptive fundamental cell should still make it efficient to search the desired crystal structures formed by those polyhedral nanobuilding blocks. Finally, it is worth noting that the ASC algorithm is also suitable to generate random packings of polyhedral particles. Crucial dynamical parameters of the system, such as the strain rate can be properly controlled to produce packings with varying degrees of disorder, including the maximally random jammed ones [51]. We will explore disordered packings in future work.

\section{ACKNOWLEDGMENTS}

We are grateful to Henry Cohn, John Conway and Alex Jaoshvili for helpful comments on our manuscript. S. T. thanks the Institute for Advanced Study for its hospitality during his stay there. This work was supported by the Division of Mathematical Sciences at the National Science Foundation under Award Number DMS-0804431 and by the MRSEC Program of the National Science Foundation under Award Number DMR-0820341. The figures showing the polyhedra were generated using the AntiPrism package developed by Adrian Rossiter. 


\section{APPENDIX A: OPTIMAL LATTICE PACKINGS OF THE PLATONIC AND ARCHIMEDEAN SOLIDS}

Here we collect fundamental packing characteristics (i.e., the lattice vectors, densities and contact numbers) of the optimal lattice packings of the Platonic and Archimedean solids, most of which are obtained from Refs. 22, 34 and 23. The Platonic solids are explicitly defined as the regions (sets of points) bounded by a set of linear equations of the coordinates. The Archimedean solids are either defined as intersections of different Platonic solids or delineated based on their symmetry. A point set $S$ representing the polyhedron multiplied by a number $\alpha$ means an isotropic expansion of the polyhedron $S$ with linear ratio $\alpha . S_{1} \cap S_{2}$ represents the intersection region of two polyhedra $S_{1}$ and $S_{2}$. The lattice vectors are given by column vectors (i.e., the basis vectors are the unit vectors along the coordinate axis). The naming code used below for the Platonic and Archimedean solids is the same one used in Figs. 1 and 3.

A tetrahedron (P1) has 4 vertices, 6 edges and 4 triangular faces. It is defined as the region

$P 1=\left\{x \in \mathbb{R}^{3}: x_{1}+x_{2}+x_{3} \leq 1,-x_{1}-x_{2}+x_{3} \leq 1,-x_{1}+x_{2}-x_{3} \leq 1, x_{1}-x_{2}-x_{3} \leq 1\right\}$.

The optimal lattice vectors are given by

$$
\mathbf{a}_{1}=(2,-1 / 3,-1 / 3)^{T}, \quad \mathbf{a}_{2}=(-1 / 3,2,-1 / 3)^{T}, \quad \mathbf{a}_{3}=(-1 / 3,-1 / 3,2)^{T},
$$

where the superscript "T" denotes transpose of a column vector. Each tetrahedron of the packing contacts 14 others. The packing density is $\phi_{\max }^{L}=18 / 49=0.367346 \ldots$

An icosahedron (P2) has 12 vertices, 30 edges and 20 triangular faces. It is defined as the region

$P 2=\left\{x \in \mathbb{R}^{3}:\left|x_{1}\right|+\left|x_{2}\right|+\left|x_{3}\right| \leq 1,\left|\Phi x_{1}\right|+\left|x_{3} / \Phi\right| \leq 1,\left|\Phi x_{2}\right|+\left|x_{1} / \Phi\right| \leq 1,\left|\Phi x_{3}\right|+\left|x_{2} / \Phi\right| \leq 1\right\}$ 
where $\Phi=(1+\sqrt{5}) / 2$ is the golden ratio. The lattice vectors are given by

$$
\begin{gathered}
\mathbf{a}_{1}(\bar{x})=\frac{2}{(1+\Phi)}\left(\begin{array}{c}
(-33 / 8-39 \sqrt{5} / 8) \bar{x}^{2}+(39 / 4+33 \sqrt{5} / 4) \bar{x}-11 / 4-3 \sqrt{5} / 2 \\
(-1 / 4-\sqrt{5} / 4) \bar{x}+1+\sqrt{5} / 2 \\
(33 / 8+39 \sqrt{5} / 8) \bar{x}^{2}+(-19 / 2-8 \sqrt{5}) \bar{x}+13 / 4+3 \sqrt{5} / 2
\end{array}\right), \\
\mathbf{a}_{2}(\bar{x})=\frac{2}{(1+\Phi)}\left(\begin{array}{c}
(-39 / 8-33 \sqrt{5} / 40) \bar{x}^{2}+(35 / 4+41 \sqrt{5} / 20) \bar{x}-5 / 2-23 \sqrt{5} / 20 \\
(5 / 4+\sqrt{5} / 4) \bar{x}-1-\sqrt{5} / 2 \\
(-39 / 8-33 \sqrt{5} / 40) \bar{x}^{2}+(15 / 2+9 \sqrt{5} / 5) \bar{x}+13 / 4-3 \sqrt{5} / 20
\end{array}\right), \\
\mathbf{a}_{3}(\overline{(}(x))=\frac{2}{(1+\Phi)}\left(\begin{array}{c}
(3 / 2+\sqrt{5} / 2) \bar{x}-2-\sqrt{5} \\
\bar{x} \\
0
\end{array}\right),
\end{gathered}
$$

where $\bar{x} \in(1,2)$ is the unique root of the polynomial

$$
1086 x^{3}-(1063+113 \sqrt{5}) x^{2}+(15 \sqrt{5}+43) x+102+44 \sqrt{5}=0 .
$$

It is found that $\bar{x}=1.59160301 \ldots$ and therefore the lattice vectors, up to nine significant figures, are given by

$$
\begin{gathered}
\mathbf{a}_{1}=(0.711782425,0.830400102,1.07585146)^{T}, \\
\mathbf{a}_{2}=(-0.871627249,0.761202911,0.985203828)^{T}, \\
\mathbf{a}_{3}=(-0.06919791,1.59160301,0)^{T} .
\end{gathered}
$$

Each icosahedron of the packing contacts 12 others. The packing density is $\phi_{\max }^{L}=$ $0.836357 \ldots$

A dodecahedron (P3) has 20 vertices, 30 edges and 12 pentagonal faces. It is defined as the region

$$
P 3=\left\{x \in \mathbb{R}^{3}:\left|\Phi x_{1}\right|+\left|x_{2}\right| \leq 1,\left|\Phi x_{2}\right|+\left|x_{3}\right| \leq 1,\left|\Phi x_{3}\right|+\left|x_{1}\right| \leq 1\right\} .
$$

The optimal lattice vectors are given by

$$
\begin{aligned}
& \mathbf{a}_{1}=(2 /(1+\Phi), 2 /(1+\Phi), 0)^{T} \\
& \mathbf{a}_{2}=(2 /(1+\Phi), 0,2 /(1+\Phi))^{T} \\
& \mathbf{a}_{3}=(0,2 /(1+\Phi), 2 /(1+\Phi))^{T}
\end{aligned}
$$


Each dodecahedron of the packing contacts 12 others. The packing density is $\phi_{\max }^{L}=$ $(2+\Phi) / 4=0.904508 \ldots$

An octahedron (P4) has 6 vertices, 12 edges and 8 triangular faces. It is defined as the region

$$
P 4=\left\{x \in \mathbb{R}^{3}:\left|x_{1}\right|+\left|x_{2}\right|+\left|x_{3}\right| \leq 1\right\} .
$$

The optimal lattice vectors are given by

$$
\mathbf{a}_{1}=(2 / 3,1,1 / 3)^{T}, \quad \mathbf{a}_{2}=(-1 / 3,-2 / 3,1)^{T}, \quad \mathbf{a}_{3}=(-1,1 / 3,-2 / 3)^{T} .
$$

Each octahedron of the packing contacts 14 others. The packing density is $\phi_{\max }^{L}=18 / 19=$ $0.947368 \ldots$

A cube (P5) has 8 vertices, 12 edges and 6 square faces. It is defined as the region

$$
P 5=\left\{x \in \mathbb{R}^{3}:\left|x_{i}\right| \leq 1\right\} .
$$

The optimal lattice vectors are given by

$$
\mathbf{a}_{1}=(2,0,0)^{T}, \quad \mathbf{a}_{2}=(0,2,0)^{T}, \quad \mathbf{a}_{3}=(0,0,2)^{T} .
$$

Each cube of the packing contacts 26 others (which includes vertex-to-vertex contacts). The packing density is $\phi_{\max }^{L}=1$.

A truncated tetrahedron (A1) has 12 vertices, 18 edges and 8 face: 4 hexagons and 4 triangles. It is defined as the region

$$
A 1=\left\{x \in \mathbb{R}^{3}: x \in 5 \cdot P 1 \cap-3 \cdot P 1\right\} .
$$

The optimal lattice vectors are given by

$$
\mathbf{a}_{1}=(4 / 3,4,8 / 3)^{T}, \quad \mathbf{a}_{2}=(4,-8 / 3,-4 / 3)^{T}, \quad \mathbf{a}_{3}=(-8 / 3,4 / 3,-4)^{T} .
$$

Each truncated tetrahedron of the packing contacts 14 others. The packing density is $\phi_{\max }^{L}=207 / 304=0.680921 \ldots$

A truncated icosahedron (A2) has 60 vertices, 90 edges and 32 faces: 20 hexagons and 12 pentagons. It is defined as the region

$$
A 2=\left\{x \in \mathbb{R}^{3}: x \in(1+\Phi) \cdot P 2 \cap(4 / 3+\Phi) \cdot P 3\right\} .
$$


The optimal lattice vectors are given by

$$
\mathbf{a}_{1}=(1+\Phi) \mathbf{a}_{1}^{P 2}, \quad \mathbf{a}_{2}=(1+\Phi) \mathbf{a}_{2}^{P 2}, \quad \mathbf{a}_{3}=(1+\Phi) \mathbf{a}_{3}^{P 2}
$$

where $\mathbf{a}_{i}^{P 2}(i=1,2,3)$ are the lattice vectors of the optimal lattice packing of icosahedra. Each truncated icosahedron of the packing contacts 12 others. The packing density is $\phi_{\max }^{L}=0.784987 \ldots$

A snub cube (A3) has 24 vertices, 60 edges and 38 faces: 6 squares and 32 triangles. Let the snub cube orientated in a way such that its 6 square faces lie in the hyperplanes

$$
\left\{x \in \mathbb{R}^{3}:\left|x_{i}\right|=1(i=1,2,3)\right\} .
$$

The optimal lattice vectors are given by

$$
\mathbf{a}_{1}=(2,0,0)^{T}, \quad \mathbf{a}_{2}=(0,0,2)^{T}, \quad \mathbf{a}_{3}=\left(1,2 / y^{*}-2,-1\right)^{T} .
$$

where $y^{*}$ is the unique real solution of $y^{3}+y^{2}+y=1$. Each snub cube of the packing contacts 12 others. The packing density is $\phi_{\max }^{L}=0.787699 \ldots$

A snub dodecahedron (A4) has 60 vertices, 150 edges and 92 faces: 12 pentagons and 80 triangles. Let the snub dodecahedron orientated in a way such that its 12 pentagonal faces lie in the hyperplanes of the faces of the dodecahedron $(1+\Phi) \cdot P 3$. The optimal lattice vectors are given by

$$
\mathbf{a}_{1}=(2,2,0)^{T}, \quad \mathbf{a}_{2}=(2,0,2)^{T}, \quad \mathbf{a}_{3}=(0,2,2)^{T} .
$$

Each snub dodecahedron of the packing contacts 12 others. The packing density is $\phi_{\max }^{L}=$ $0.788640 \ldots$

A rhombicosidodecahdron (A5) (also known as small rhombicosidodecahedron) has 60 vertices, 120 edges and 62 face: 12 pentagons, 30 squares and 20 triangles. It is defined as the region:

$$
A 5=\left\{x \in \mathbb{R}^{3}: x \in(3 \Phi+2) \cdot[P 5 \cap(1+\sqrt{2}) P 4] \cap(4 \Phi+1) \cdot P 2 \cap(3+3 \Phi) \cdot P 3\right\} .
$$

The optimal lattice vectors are given by

$$
\begin{aligned}
& \mathbf{a}_{1}=((\Phi-1) /(2 \Phi+1), 7,(9 \Phi+4) /(2 \Phi+1))^{T}, \\
& \mathbf{a}_{2}=((9 \Phi+4) /(2 \Phi+1),(\Phi-1) /(2 \Phi+1), 7)^{T}, \\
& \mathbf{a}_{3}=(7,(9 \Phi+4) /(2 \Phi+1),(\Phi-1) /(2 \Phi+1))^{T} .
\end{aligned}
$$


Each rhombicosidodecahedron of the packing contacts 12 others. The packing density is $\phi_{\max }^{L}=(8 \Phi+46) /(36 \Phi+15)=0.804708 \ldots$

A truncated icosidodecahdron (A6) (also known as great rhombicosidodecahedron) has 120 vertices, 180 edges and 62 faces: 12 decagons, 20 hexagons and 30 squares. It is defined as the region

$$
A 6=\left\{x \in \mathbb{R}^{3}: x \in(5 \Phi+4) \cdot[P 5 \cap(1+\sqrt{2}) P 4] \cap(6 \Phi+3) \cdot P 2 \cap(5+5 \Phi) \cdot P 3\right\} .
$$

The optimal lattice vectors are given by

$$
\mathbf{a}_{1}=(10,10,0)^{T}, \quad \mathbf{a}_{2}=(10,0,10)^{T}, \quad \mathbf{a}_{3}=(0,10,10)^{T}
$$

Each truncated icosidodecahedron of the packing contacts 12 others. The packing density is $\phi_{\max }^{L}=(2 \Phi / 5+9 / 50)=0.827213 \ldots$

A truncated cubeoctahedron (A7) (also known as great rhombicubeoctahedron) has 48 vertices, 72 edges and 26 faces: 6 octagons, 8 hexagons and 12 squares. It is defined as the region

$$
\begin{array}{r}
A 7=\left\{x \in \mathbb{R}^{3}:\left|x_{1}\right|+\left|x_{2}\right| \leq(2+3 \sqrt{2}),\left|x_{2}\right|+\left|x_{3}\right| \leq(2+3 \sqrt{2}),\right. \\
\left.\left|x_{2}\right|+\left|x_{3}\right| \leq(2+3 \sqrt{2}), x \in(2 \sqrt{2}+1) \cdot P 5 \cap(3 \sqrt{2}+3) \cdot P 4\right\} .
\end{array}
$$

The optimal lattice vectors are given by

$$
\begin{gathered}
\mathbf{a}_{1}=(4 \sqrt{2}+2,-4 \sqrt{2}-1+2 \alpha, 4 \sqrt{2}+1-2 \alpha)^{T}, \\
\mathbf{a}_{2}=(\sqrt{2} / 2-3 / 2+\alpha,-3 \sqrt{2} / 2+1 / 2+\alpha, 4 \sqrt{2}+2)^{T}, \\
\mathbf{a}_{3}=(7 / 2+7 \sqrt{2} / 2-\alpha, 1+2 \alpha, 5 \sqrt{2} / 2+3 / 2-\alpha)^{T},
\end{gathered}
$$

where $\alpha=\sqrt{33}(\sqrt{2}+1) / 6$. Each truncated cubeoctahedron of the packing contacts 12 others. The packing density is $\phi_{\max }^{L}=0.8493732 \ldots$..

An icosidodecahedron (A8) has 30 vertices, 60 edges and 32 faces: 12 pentagons and 20 triangles. It is defined as the region

$$
A 8=\left\{x \in \mathbb{R}^{3}: x \in P 2 \cap P 3\right\}
$$

The optimal lattice vectors are given by

$$
\begin{aligned}
& \mathbf{a}_{1}=(2 /(1+\Phi), 2 /(1+\Phi), 0)^{T} \\
& \mathbf{a}_{2}=(2 /(1+\Phi), 0,2 /(1+\Phi))^{T} \\
& \mathbf{a}_{3}=(0,2 /(1+\Phi), 2 /(1+\Phi))^{T}
\end{aligned}
$$


Each icosidodecahedron of the packing contacts 12 others. The packing density is $\phi_{\max }^{L}=$ $(14+17 \Phi) / 48=0.864720 \ldots$

A rhombicuboctahedron (A9) (also known as small rhombicubeoctahedron) has 24 vertices, 48 edges and 26 faces: 18 squares and 8 triangles. It is defined as the region

$$
\begin{array}{r}
A 9=\left\{x \in \mathbb{R}^{3}:\left|x_{1}\right|+\left|x_{2}\right| \leq 2,\left|x_{2}\right|+\left|x_{3}\right| \leq 2,\right. \\
\left.\left|x_{1}\right|+\left|x_{3}\right| \leq 2, x \in \sqrt{2} \cdot P 5 \cap(4-\sqrt{2}) \cdot P 4\right\} .
\end{array}
$$

The optimal lattice vectors are given by

$$
\mathbf{a}_{1}=(2,2,0)^{T}, \quad \mathbf{a}_{2}=(2,0,2)^{T}, \quad \mathbf{a}_{3}=(0,2,2)^{T} .
$$

Each truncated cubeoctahedron of the packing contacts 12 others. The packing density is $\phi_{\max }^{L}=(16 \sqrt{2}-20) / 3=0.875805 \ldots$

A truncated dodecahedron (A10) has 60 vertices, 90 edges and 32 faces: 12 decagons and 20 triangles. It is defined as the region

$$
A 10=\{x \in(1+\Phi) \cdot P 3 \cap[(7+12 \Phi) /(3+4 \Phi)] \cdot P 2\} .
$$

The optimal lattice vectors are given by

$$
\mathbf{a}_{1}=(2,2,0)^{T}, \quad \mathbf{a}_{2}=(2,0,2)^{T}, \quad \mathbf{a}_{3}=(0,2,2)^{T} .
$$

Each truncated dodecahedron of the packing contacts 12 others. The packing density is $\phi_{\max }^{L}=(5 \Phi+16) /(24 \Phi-12)=0.897787 \ldots$

A cuboctahedron (A11) has 12 vertices, 24 edges and 14 faces: 6 squares and 8 triangles. It is defined as the region

$$
A 11=\{x \in P 5 \cap 2 \cdot P 4\} .
$$

The optimal lattice vectors are given by

$$
\mathbf{a}_{1}=(2,-1 / 3,-1 / 3)^{T}, \quad \mathbf{a}_{2}=(-1 / 3,2,-1 / 3)^{T}, \quad \mathbf{a}_{3}=(-1 / 3,-1 / 3,2)^{T} .
$$

Each cuboctahedron of the packing contacts 14 others. The packing density is $\phi_{\max }^{L}=$ $45 / 49=0.918367 \ldots$

A truncated cube (A12) has 24 vertices, 36 edges and 14 faces: 6 octagons and 8 triangles. It is defined as the region

$$
A 12=\{x \in P 5 \cap(1+\sqrt{2}) \cdot P 4\} .
$$


The optimal lattice vectors are given by

$$
\mathbf{a}_{1}=(2,-2 \alpha, 0)^{T}, \quad \mathbf{a}_{2}=(0,2,-2 \alpha)^{T}, \quad \mathbf{a}_{3}=(-2 \alpha, 0,2)^{T},
$$

where $\alpha=(2-\sqrt{2}) / 3$. Each truncated cube of the packing contacts 14 others. The packing density is $\phi_{\max }^{L}=9 /(5+3 \sqrt{2})=0.973747 \ldots$

A truncated octahedron (A13) has 24 vertices, 36 edges and 14 faces: 8 hexagons and 6 squares. It is defined as the region

$$
A 13=\{x \in P 5 \cap(3 / 2) \cdot P 4\} .
$$

The optimal lattice vectors are given by

$$
\mathbf{a}_{1}=(2,0,0)^{T}, \quad \mathbf{a}_{2}=(2,2,0)^{T}, \quad \mathbf{a}_{3}=(1,1,-1)^{T} .
$$

Each truncated octahedron of the packing contacts 14 others. The packing density is $\phi_{\max }^{L}=$ 1.

\section{APPENDIX B: LATTICE VECTORS AND OTHER CHARACTERISTICS OF THE DENSEST KNOWN TETRAHEDRAL PACKING}

Here we report the lattice vectors of fundamental cell for the densest known tetrahedral packing with 314 particles up to 12 significant figures, although our numerical precision is not limited by that. The side length of the tetrahedron is $d_{0}=1.8$ and its volume is $v_{p}=\sqrt{2} d_{0}^{3} / 12=0.687307791313$. The lattice vectors are given by

$$
\begin{gathered}
\mathbf{a}_{1}=(6.130348985438,-1.714011427194,0.022462185673)^{T} \\
\mathbf{a}_{2}=(1.622187720300,6.106123418234,0.123567805838)^{T} \\
\mathbf{a}_{3}=(0.055014355972,0.116436714923,6.526427521512)^{T}
\end{gathered}
$$

The volume of the fundamental cell is $\operatorname{Vol}(F)=\left|\mathbf{a}_{1} \times \mathbf{a}_{2} \cdot \mathbf{a}_{3}\right|=262.344828467328$. Thus, the packing density is readily computed as

$$
\phi=\frac{N \cdot v_{p}}{\operatorname{Vol}(F)}=\frac{314 \times 0.687307791313}{262.344828467328}=0.822637319490 .
$$

The coordinates of each of the 314 tetrahedra are given elsewhere [37]. 


\section{APPENDIX C: LATTICE VECTORS OF THE DENSEST KNOWN PACKING OF TRUNCATED TETRAHEDRA}

The "primitive Welsh" tessellation of space consists of truncated large regular tetrahedra and small regular tetrahedra, as described in Ref. 13. When the small tetrahedra are removed, the remaining truncated tetrahedra give the densest known periodic (non-lattice) packing of such objects. Following Ref. 13, the centroids of the truncated tetrahedra sit at the nodes $\mathbf{0}$ and $\mathbf{1}$ of the two adjacent cells of a body-centered cubic lattice shown in Fig. 2(a) of Ref. 13. Let length of the edges of the truncated tetrahedron be $d_{E}=\sqrt{2} / 2$, then the lattice vectors are given by

$$
\mathbf{a}_{1}=(1,1,0)^{T}, \quad \mathbf{a}_{2}=(1,0,1)^{T}, \quad \mathbf{a}_{3}=(0,1,1)^{T} .
$$

The basis vectors for the centroids of $\mathbf{0}$-type and $\mathbf{1}$-type truncated tetrahedra are respectively given by

$$
\mathbf{b}_{0}=(1 / 2,1 / 2,1 / 2)^{T}, \quad \mathbf{b}_{1}=(0,0,0)^{T}
$$

[1] J. D. Bernal, in Liquids: Structure, Properties, Solid Interactions, (Ed. T. J. Hughel, Elsevier, New York, 1965).

[2] R. Zallen, The Physics of Amorphous Solids (Wiley, New York, 1983).

[3] S. Torquato, Random Heterogeneous Materials: Microstructure and Macroscopic Properties (Springer-Verlag, New York, 2002).

[4] P. M. Chaikin and T. C. Lubensky, Principles of Condensed Matter Physics (Cambridge University Press, New York, 2000).

[5] S. F. Edwards, Granular Matter (Ed. A. Mehta, Springer-Verlag, New York, 1994); E. R. Nowak, J. B. Knight, E. Ben-Naim, H. M. Jaeger, and S. R. Nagel, Phys. Rev. E 57, 1971 (1998); R. P. Behringer, Nature 435, 1079 (2005); M. Schröter, D. I. Goldman, and H. L. Swinney, Phys. Rev. E 71, 030301 (2005).

[6] I. C. Kim and S. Torquato, J. Appl. Phys. 69, 2280 (1991); D. Cule and S. Torquato, Phys. Rev. B 58, R11829 (1998); M. C. Rechtsman and S. Torquato, J. Appl. Phys. 103, 084901 (2008); T. Zohdi, Int. J. Numer. Meth. Eng. 76, 1250 (2008). 
[7] J. H. Conway and N. J. A. Sloane, Sphere Packings, Lattices and Groups (Springer-Verlag, New York, 1998).

[8] H. Cohn and N. Elkies, Ann. Math. 157, 689 (2003); H. Cohn, Geom. Topol. 6, 329 (2002).

[9] G. Parisi and F. Zamponi, J. Stat. Mech.: Theory Exp. 2006, P03017. S. Torquato and F. H. Stillinger, Phys. Rev. E 73, 031106 (2006); S. Torquato, O. U. Uche and F. H. Stillinger, Phys. Rev. E 74, 061308 (2006).

[10] T. C. Hales, Ann. Math. 162, 1065 (2005).

[11] A. Bezdek and W. Kuperberg, in DIMACS Series in Discrete Math, Theoret. Comput. Sci. 4 (Eds. P. Gritzmann and B. Sturmfels, AMS, Providence, RI, 1991); J. M. Wills, Mathematika 38, 318 (1991).

[12] A. Donev, F. H. Stillinger, P. M. Chaikin, and S. Torquato, Phys. Rev. Lett. 92, 255506 (2004).

[13] J. H. Conway and S. Torquato, Proc. Nat. Acad. Sci. 103, 10612 (2006).

[14] P. Chaikin, S. Wang, and A. Jaoshvili, APS March Meeting Abstract (2007).

[15] E. R. Chen, Discrete Comput. Geom. 40, 214 (2008).

[16] Y. Jiao, S. H. Stillinger, and S. Torquato, Phys. Rev. Lett. 100, 245504 (2008).

[17] Y. Jiao, S. H. Stillinger, and S. Torquato, Phys. Rev. E 79, 041309 (2009).

[18] Dense polyhedral packings have relevance to molecular crystal structures of organic compounds. See, for example, A. I. Kitaigorodskii, Molecular Crystal and Molecules (Academic, New York, 1973).

[19] It is well known that viral capsids have icosahedral symmetry. See, for example; R. Zandi, D. Reguera, R. F. Bruinsma, W. M. Gelbart, and 5. J. Rudnick, Proc. Nat. Acad. Sci. 101, 15556 (2004).

[20] A. R. Kansal, S. Torquato, and F. H. Stillinger, Phys. Rev. E 66, 041109 (2002). This tilingdegeneracy example vividly illustrates a fundamental point made in Ref. 20, namely, packing arrangements of nonoverlapping objects at some fixed density can exhibit a large variation in their degree of structural order.

[21] In the physical sciences and engineering, this is referred to as a Bravais lattice. Unless otherwise stated, the term "lattice" will refer to a Bravais lattice only, not the more general classification of a periodic lattice with a basis.

[22] H. Minkowski, Nachr. K. Ges. Wiss. Göttingen, Math.-Phys. KL, 311 (1904). 
[23] U. Betke and M. Henk, Comput. Geom. 16, 157 (2000).

[24] The overlap potential function of a pair of strictly convex and smooth particles is an analytic function of the positions, orientations and shapes of the two particles, whose value indicates whether the two particles overlap or not, or whether they are tangent to one another; see also Ref. 25.

[25] A. Donev. S. Torquato and F. H. Stillinger, J. Comput. Phys. 202, 737 (2005).

[26] A. Donev. S. Torquato and F. H. Stillinger, J. Comput. Phys. 202, 765 (2005).

[27] S. Torquato and Y. Jiao, Nature, 460876 (2009).

[28] Note that in Fig. 1 of Ref. [27], "P6" should be "A1."

[29] In this paper, a deformation refers to a volume-preserving shape change of the fundamental cell (i.e., a shear), which is to be distinguished from a volume change of the cell, which can either be a compression or an expansion of the cell.

[30] S. Torquato and Y. Jiao, unpublished.

[31] W. S. Jodrey and E. M. Tory, Phys. Rev. A 32, 2347 (1985); M. D. Rintoul and S. Torquato, Phys. Rev. E 58, 532 (1998); O. U. Uche, F. H. Stillinger, and S. Torquato, Physica A 342, 428 (2004); A. Donev, J. Burton, F. H. Stillinger and S. Torquato, Phys. Rev. B 73, 054109 (2006).

[32] S. Torquato and F. H. Stillinger, J. Phys. Chem. B, 105, 11849 (2001); S. Torquato, A. Donev, and F. H. Stillinger, Int. J. Solids Struct. 40, 7143 (2003); A. Donev, S. Torquato, F. H. Stillinger and R. Connelly, J. Appl. Phys. 95, 989 (2004).

[33] E. G. Golshtein and N. V. Tretyakov, Modified Lagrangians and Monotone Maps in Optimization (Wiley, New York, 1996).

[34] D. J. Hoylman, Bull. Am. Math. Soc. 76, 135 (1970).

[35] In Ref. 13, the authors suggested (not conjectured) that the regular tetrahedron "may not be able to pack as densely as the sphere, which would contradict a conjecture of Ulam." Indeed, the results of Ref. 15 verifies that the tetrahedron does not violate Ulam's conjecture.

[36] A. Donev, R. Connelly, F. H. Stillinger and S. Torquato, Phys. Rev. E 75, 051304 (2007).

[37] The supplementary materials can be found at http://cherrypit.princeton.edu/pre-packings.html.

[38] The fundamental cells of the initial configurations for the optimization algorithm involve periodic copies of the packings listed in Table I. Specifically, if the lattice vectors of the packings listed in Table $\mathrm{I}$ are denoted by $\mathbf{e}_{1}, \mathbf{e}_{2}$ and $\mathbf{e}_{3}$, then the lattice vectors associated 
with the initial configurations are given by $\mathbf{e}_{1}^{L}=n_{1} \mathbf{e}_{1}, \mathbf{e}_{2}^{L}=n_{2} \mathbf{e}_{2}$ and $\mathbf{e}_{3}^{L}=n_{3} \mathbf{e}_{3}$, where $n_{1}, n_{2}, n_{3} \geq 1$ are integers. Thus, the number of tetrahedra in the fundamental cells of the initial configurations is an integral multiple, i.e., $n_{1} \times n_{2} \times n_{3}$, of that for the corresponding packings given in Table I. For the optimal lattice packing, $n_{1}=n_{2}=n_{3}=3$; for the uniform packing, $n_{1}=n_{2}=n_{3}=3$; for the Welsh packing, $n_{1}=n_{2}=n_{3}=1$; for the icosahedral packing, $n_{1}=n_{2}=n_{3}=1$; and for the wagon-wheels packing, $n_{1}=n_{2}=2, n_{3}=1$.

[39] This bound was motivated by a question posed by Robert Connelly in May, 2005 at a Banff International Research Station Workshop entitled "Densest Packings of Spheres," organized by K. Bezdek, H. Cohn and C. Radin. Specifically, he asked whether an upper bound on the maximal density of congruent ellipsoid packings in $\mathbb{R}^{3}$ that is less than unity for any aspect ratios of the ellipsoid could be formulated. One of us in attendance (S. T.) provided the version of upper bound (14) for the special case of ellipsoids. Of course, for ellipsoids, the bound (14) is less than unity provided that the eccentricity of the ellipsoid is sufficiently small. However, here we recognize that the same type of upper bound can yield a reasonably tight upper bound for other nonspherical shapes provided that the particle has a small asphericity $\gamma$.

[40] Although we cannot exclude the possibility that there exist other optimal packing particle arrangements that possess the same density as the optimal lattice packings, it is highly unlikely that those arrangements could be constructed in the same way way as the stacking variants of the optimal FCC lattice packings for spheres (the so-called Barlow packings). The polyhedral shape of the particles does not allow them to fit snugly into the "holes" formed in the particle layers above and below a particular layer, as in the case of spheres, and hence stackings of the layers cannot result in packings with densities that are higher than that of the optimal lattice packing. If degenerate optimal packings exist, they cannot be of the "Barlow" type.

[41] L. Fejes Tóth, Regular Figures (Macmillan, New York, 1964).

[42] M. Gardner, The Colossal Book of Mathematics: Classic Puzzles, Paradoxes, and Problems (Norton, New York, 2001), p. 13.

[43] A. Bezdek, Intuitive Geometry 63, 17 (1994); T. C. Hales, Discrete Comput. Geom. 36, 5 (2006).

[44] P. R. Cromwell, Polyhedra (Cambridge University Press, 1997).

[45] H. S. M. Coxeter, Regular Polytopes (Dover, New York, 1973).

[46] S. Torquato and F. H. Stillinger, Exp. Math. 15, 307 (2006); A. Scardicchio, F. H. Stillinger 
and S. Torquato, J. Math. Phys. 49, 043301 (2008).

[47] E. C. Creyson, J. E. Barton and T. W. Odom, Small 2, 368 (2006).

[48] Y. G. Sun and Y. N. Xia, Science 298, 2176 (2002)

[49] T. S. Ahmadi, Z. L. Wang, T. C. Green, A. Henglein and M. A. El-Sayed, Science 272, 1924 (1996).

[50] R. Jin, Y. Cao, C. A. Mirkin, K. L. Kelly, G. C. Schatz and J. G. Zeng, Science 294, 1901 (2001).

[51] S. Torquato, T. M. Truskett and P. G. Debenedetti, Phys. Rev. Lett. 84, 2064 (2000). 